\title{
Evaluation of Patients with Insufficient Efficacy and/ or Tolerability to Triptans for the Acute Treatment of Migraine: A Systematic Literature Review
}

\author{
Elizabeth Leroux · Andrew Buchanan (D) - Louise Lombard • \\ Li Shen Loo · Daisy Bridge · Ben Rousseau • Natasha Hopwood • \\ Brandy R. Matthews · Uwe Reuter
}

Received: June 17, 2020 / Accepted: September 2, 2020 / Published online: September 29, 2020

(C) The Author(s) 2020

\section{ABSTRACT}

Introduction: Use of triptans for acute treatment of migraine is associated with insufficient efficacy and/or tolerability in approximately $30-40 \%$ of people. We conducted a systematic literature review (SLR) to synthesize definitions, terminology, subsequent treatment outcomes, and characteristics associated with this subpopulation.

Methods: A comprehensive SLR was conducted to identify studies, published from Jan 1995 to

Digital Features To view digital features for this article go to https://doi.org/10.6084/m9.figshare.12902390.

Electronic supplementary material The online version of this article (https://doi.org/10.1007/s12325020-01494-9) contains supplementary material, which is available to authorized users.

E. Leroux

Brunswick Medical Center, Montréal, QC, Canada

A. Buchanan $(\bowtie) \cdot$ L. Lombard · L. S. Loo ·

B. R. Matthews

Eli Lilly and Company, Indianapolis, IN, USA

e-mail: buchanan_andrew_s@lilly.com

D. Bridge · B. Rousseau · N. Hopwood

Adelphi Values, Bollington, Cheshire, UK

U. Reuter

Department of Neurology, Charité

Universitätsmedizin Berlin, Berlin, Germany
May 2019, which focused on insufficient efficacy and/or tolerability to triptans.

Results: Thirty-five publications were identified, of which 22 described randomized controlled trials and open-label studies, and 13 described observational studies. Across studies, multiple objectives and a high amount of variability in methodologies and outcomes were noted. The most commonly applied measures of efficacy were headache pain freedom and pain relief at $2 \mathrm{~h}$. Ten studies assessed efficacy of switching or optimizing treatment in patients with historical insufficient efficacy or tolerability to previous triptan treatment and demonstrated varying levels of success. Factors associated with increased risk of triptan insufficient efficacy included severe baseline headache severity, photophobia, phonophobia, nausea, and depression.

Conclusions: Irrespective of the methodology or definition used to identify people with insufficient efficacy and/or tolerability to triptans, study results support the assertion that a high unmet need remains for effective acute treatment of migraine.

Keywords: Acute treatment; Insufficient efficacy and/or tolerability to triptans; Migraine; Triptans; Triptan non-responders; Triptan response 


\section{Key Summary Points}

This systematic literature review identified substantial variability across studies in definitions and methodologies used to identify insufficient efficacy and/or tolerability to triptans for acute treatment of migraine.

Across studies, the most commonly used outcomes to measure efficacy were pain relief and pain freedom at $2 \mathrm{~h}$.

The totality of evidence suggests that a proportion of patients with insufficient efficacy and/or tolerability to one triptan may benefit from switching to a different triptan.

Factors associated with increased risk of insufficient efficacy and/or tolerability to triptans include severe baseline headache severity, photophobia, phonophobia, nausea, and depression.

Findings from this review suggest that a large unmet need remains for people with insufficient efficacy and/or tolerability to triptans, irrespective of the definitions or methodologies used to identify this population.

\section{DIGITAL FEATURES}

This article is published with digital features to facilitate understanding of the article. To view digital features for this article go to https://doi. org/10.6084/m9.figshare.12902390.

\section{INTRODUCTION}

Since their introduction in the 1990s, triptans have been considered therapy of choice for the acute treatment of moderate to severe migraine attacks [1]. Triptans act primarily on $5-\mathrm{HT}_{1 \mathrm{~B}} / 5-$ $\mathrm{HT}_{1 \mathrm{D}}$ receptors which are present in multiple regions including cerebral blood vessels, sensory trigeminal nerves, and trigeminal nucleus caudalis neurons [2]. Activation of these receptors leads to inhibition of release of pro-inflammatory neuropeptides and vasoconstriction [3]. Triptans have been available to patients in the USA for more than 20 years; estimates from a longitudinal US study that evaluated utilization and reimbursement trends point to an increase in triptan prescriptions from 87,348 in 1993 to 1.2 million in 2013 [4]. Globally, triptan use is more challenging to study, in part because of limited data availability, and partly because triptans are available over-the-counter (OTC) in some countries such as the UK, Germany, New Zealand, and Sweden [5-7].

It is estimated that approximately $30-40 \%$ of people with migraine are not successfully treated using a triptan, for reasons of insufficient efficacy and/or tolerability [8-10]. Factors including route of administration and time from onset of migraine attack to administration have been shown to influence patients' response to triptans $[11,12]$. While methods to assess response vary across studies, it is generally acknowledged that insufficient efficacy and/or tolerability to one triptan does not necessarily predict outcomes with a different triptan. The true population of patients with insufficient efficacy and/or tolerability to triptans is challenging to characterize given the complexity and high level of variability reported across patients, attacks (even within the same person), and treatments $[8,12]$. In addition, definitions applied to identify people with triptan insufficient efficacy and/or tolerability vary substantially among studies.

Given the need to better understand this population of patients with migraine and insufficient efficacy and/or tolerability to triptans, we conducted a systematic literature review (SLR) to identify randomized controlled trials (RCTs), open-label studies, and observational studies that focused on evaluating any aspect of this population. Our objectives were to investigate outcomes associated with switching or optimizing acute treatments; to determine definitions and terminologies used to identify and describe this population; and to investigate patient, disease, attack, and treatment 
characteristics associated with this population. Robust reviews, meta-analyses, and expert clinical perspectives relevant to this topic have been previously published $[8,9,12-14]$. However, to our knowledge this is the first SLR with a broad scope specific to this population. This information is increasingly relevant as new classes of acute treatments will become options for patients who have insufficient efficacy, tolerability, or contraindications to triptans.

\section{METHODS}

\section{Data Sources and Searches}

We searched the literature for all relevant RCTs and observational studies of patients with migraine who have been treated with triptans. Using the OVID platform, we searched the following databases: EMBASE, Ovid MEDLINE ${ }^{\circledR}$ and Epub Ahead of Print, In-Process and Other Non-Indexed Citations, Daily and Versions ${ }^{\circledR}$, EBM Reviews (Cochrane Central Register of Controlled Trials, Cochrane Database of Systematic Reviews, Database of Abstracts of Reviews of Effect), PsychINFO, and EconLit. Search strategies are included in Supplementary Material.

The search strategies utilized a combination of free text searching and subject headings. Vocabulary and syntax were adjusted across databases. We also searched for any additional references by hand-searching bibliographies of relevant articles. Grey literature was carefully explored for any relevant publications since 2017 to present, utilizing clinical trial registries (ClinicalTrials.gov and WHO Internal Clinical Trials Registry Platform) and conference proceedings (Migraine Trust International Symposium [MTIS], Congress of the International Headache Society [IHC], American Headache Society [AHS] Annual Scientific Meeting, European Headache Federation [EHF] Congress, and American Academy of Neurology [AAN] Annual Meeting).

This article is based on previously conducted studies and does not contain any studies with human participants or animals performed by any of the authors.

\section{Study Selection}

Studies were included on the basis of Population, Intervention, Comparator, Outcome, Study design (PICOS) criteria for inclusion and exclusion. Studies published from Jan 1995 to May 2019 which included interventions with triptans versus any comparators and focused on insufficient efficacy and/or tolerability to triptans were screened.

\section{Screening Process and Data Extraction}

The systematic review process followed guidelines from the Cochrane Handbook of Systematic Reviews of Interventions (CHSRI) [15]. A researcher (DJ) executed the search strategies in each database and exported the results for abstract screening. In the first phase of screening, two independent analysts ( $\mathrm{NH}$ and JS) reviewed the titles and abstracts of all retrieved publications. Following this, the same two analysts independently examined full-text publications. Any discrepancies were resolved through discussion or through the involvement of a senior reviewer.

Data extraction from eligible studies, utilizing the data extraction form, was performed by one analyst and checked by another analyst against the source publication. Any disagreements in the assessment of these data were resolved by a senior reviewer.

\section{Bias Assessment and Quality Control}

Two analysts independently performed quality assessments of RCTs and observational studies. RCTs were assessed for risk of bias using the CHSRI tool [15]. Non-randomized studies were assessed for bias using the Newcastle-Ottawa Scale (NOS) [16].

Following this step, two independent analysts assessed the quality of RCT publications using the National Institute of Health and Care Excellence (NICE) checklist [17]; for observational studies, the Strengthening the Reporting of Observational Studies in Epidemiology (STROBE) checklist was used [18]. This study was conducted in accordance with the Preferred 
Reporting Items for Systematic Reviews and Meta-analysis (PRISMA) 2009 guidelines [19]. The PRISMA checklist is provided in the Supplemental Material.

\section{Definitions for Pain Freedom and Pain Relief}

When referencing efficacy response throughout this review, "pain freedom" refers to complete elimination of pain at endpoint. Unless otherwise stated, "pain relief" generally refers to a decrease in headache pain severity from moderate or severe at baseline to mild or none at endpoint using a 4-point headache severity scale.

\section{RESULTS}

\section{Overall Search Results}

The initial literature search yielded 4663 abstracts and 382 sources of grey literature. Following screening of abstracts, 45 full-text publications were reviewed, of which 31 publications met PICOS criteria for inclusion (Supplemental Material, Supplementary Table S1) in the qualitative synthesis. Four grey literature records were included in the qualitative synthesis, resulting in a total of 35 publications (Fig. 1). Risk of bias and quality control assessments were performed on 18 RCTs and seven observational studies; results are summarized in Supplemental Material. Overall, 16 RCT publications were judged to have a low or unclear risk of bias, while two had serious risk. Observational studies were judged to have mild or moderate risk of bias, except for one which was judged to have serious risk. The quality control assessments demonstrated that the source population was well described in 14 publications, inclusion/exclusion criteria were clearly detailed in 16 publications, interventions and comparators were adequately described in all studies except one, and relevant outcomes were assessed in all publications.

In several of the studies identified in this review, the definition for prior insufficiency to triptans was not specific either to only efficacy or only tolerability; the definitions generally could have applied to either or both. Therefore, we used the terminology of insufficient "efficacy and/or tolerability" throughout except in those cases where the definition was clearly attributed to one or the other.

\section{Study Characteristics and Objectives}

Of the 35 publications that met criteria for the current analysis, 16 comprised results from randomized, double-blind, placebo-controlled trials; six from open-label studies; and 13 publications disclosed results from observational studies. High-level study overviews with study designs, patient populations, interventions, and outcomes specific to the scope of this review are summarized in Tables 1 and 2. Broader and more detailed descriptions and outcomes of the studies are provided in Supplemental Material (Supplementary Tables S2 and S3). Definitions and terminology used to identify and describe insufficient efficacy and/or tolerability to trip$\tan$ across the studies are described in Table 3 for RCTs and open-label studies, and in Table 4 for observational studies. Brief summaries of important study characteristics identified within this SLR, with an emphasis on intervention studies are provided.

\section{Terminology and Definitions}

The most commonly applied terms to describe people with triptan insufficient efficacy and/or tolerability involved variations of non-response and variations of insufficient response (Tables 3 , 4). Lesser used terms involved dissatisfaction and failure to respond. Most studies relied on patients' self-reports that were largely subjective and allowed for aspects of both self-defined efficacy and tolerability. In studies with definitions that included specific efficacy parameters, pain freedom at $2 \mathrm{~h}$ and pain relief at $2 \mathrm{~h}$ were the most commonly applied criteria; some of those further designated sustained efficacy or efficacy in two out of three attacks. A small number of studies applied discontinuation of a 


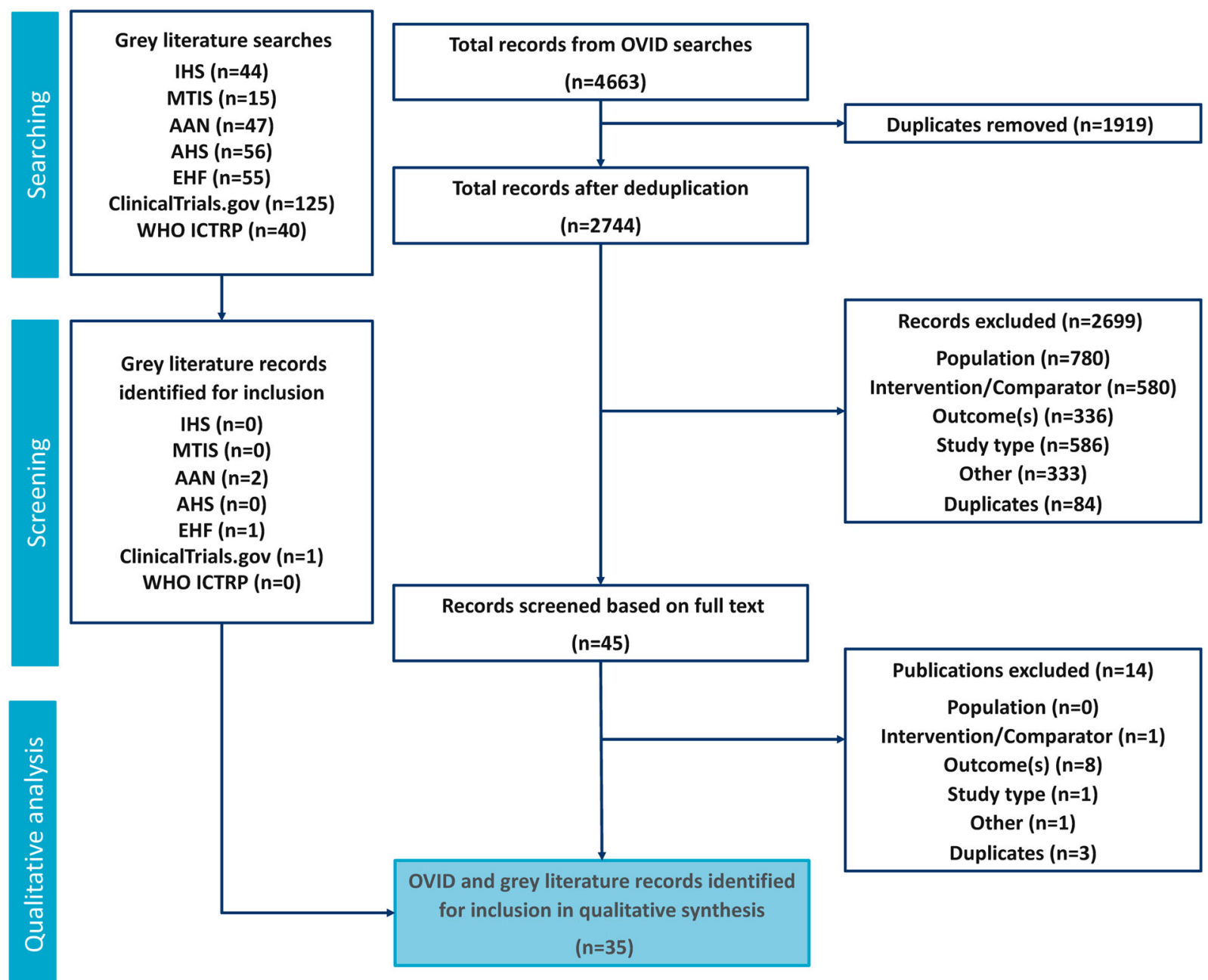

Fig. 1 PRISMA diagram ${ }^{\mathrm{a}}$. ${ }^{\mathrm{a}}$ Records retrieved from the Ovid searches included 2227 abstracts from EMBASE, 1073 from MEDLINE, 911 from EBM reviews, 450 from PyschINFO, and 2 from EconLit. AAN American Academy of Neurology, AHS American Headache Society, EHF European Headache Federation, IHS International

triptan for reasons of either efficacy or tolerability as criteria.

\section{Switching and Treatment Optimization Study Overview}

There were ten switching or treatment optimization studies which were specifically designed to assess efficacy of subsequent acute treatments in patients with historical insufficient efficacy and/or tolerability to triptans
Headache Society, MTIS Migraine Trust International Symposium, WHO ICTRP World Health Organization International Clinical Trials Registry Platform, PICOS Population, Intervention, Comparator, Outcomes, Study design

(Table 1). Of these ten studies, seven studied patients with prior sumatriptan [20-26]; one study each studied prior rizatriptan [27], prior treatment with any orally administered triptan [28], and prior treatment with short-acting orally administered triptans [29]. Key efficacy results with subsequent intervention treatments are summarized in Table 1.

Only three of the ten studies screened and validated self-reported prior triptan insufficient efficacy and/or tolerability using an open-label or single-blind run-in phase [20, 21, 23]. In 
Table 1 Study characteristics and key results of blinded randomized controlled trials and open-label studies.

\begin{tabular}{|c|c|c|c|c|}
\hline Author, year & Study design & $\begin{array}{l}\text { Population investigated/key } \\
\text { response criteria for inclusion }\end{array}$ & Intervention investigated & Key results \\
\hline \multicolumn{5}{|c|}{$\begin{array}{l}\text { Patients with historical }{ }^{a} \text { triptan insufficient efficacy and/or tolerability: subsequent response to different triptan or different triptan dose/formulation } \\
\quad(n=10)\end{array}$} \\
\hline $\begin{array}{l}\text { Seeburger, } \\
2011[20]\end{array}$ & $\begin{array}{l}\text { Randomized, blinded, } \\
\text { crossover }\end{array}$ & $\begin{array}{l}\text { Previous insufficient efficacy and/ } \\
\text { or tolerability to sumatriptan }{ }^{\mathrm{a}} \\
\text { Within-trial screening criteria } \\
\text { for insufficient efficacy: non- } \\
\text { response to sumatriptan } 100 \mathrm{mg} \\
\text { in one migraine attack eligible to } \\
\text { enter double-blind treatment } \\
\text { Within-trial screening results: } \\
\text { sumatriptan non-responsive: } \\
n=109 / 159 \text {; responsive: } \\
n=44 / 159\end{array}$ & $\begin{array}{l}\text { Rizatriptan } \\
\text { Rizatriptan } 10 \text { mg vs. placebo was } \\
\text { assessed over three migraine } \\
\text { attacks; } 109 \text { patients } \\
\text { randomized 1:1:1 to dosing } \\
\text { schedule of rizatriptan/ } \\
\text { rizatriptan/placebo, } \\
\text { rizatriptan/placebo/rizatriptan, } \\
\text { or placebo/rizatriptan/ } \\
\text { rizatriptan; } 102 \text { participants } \\
\text { treated at least one migraine } \\
\text { attack, } 98 \text { treated all three } \\
\text { attacks }\end{array}$ & $\begin{array}{l}\text { Pain relief } \mathbf{2} \text { h (across } 3 \text { attacks): } \\
\text { rizatriptan: } 51 \% \text {, placebo: } 20 \% \\
\text { Pain-free } \mathbf{2} \text { h (across } 3 \text { attacks): } \\
\text { rizatriptan: } 22 \% \text {, placebo: } 12 \% \text {. } \\
\text { (Number of treated patients } \\
102 \text { ) }\end{array}$ \\
\hline $\begin{array}{l}\text { Stark, } \\
2000[23]\end{array}$ & $\begin{array}{l}\text { Randomized, blinded, } \\
\text { parallel }\end{array}$ & $\begin{array}{l}\text { Previous insufficient efficacy and/ } \\
\text { or tolerability to sumatriptan } \\
\text { W } \\
\text { Within-trial screening criteria } \\
\text { for insufficient efficacy (single- } \\
\text { blind assessment): non- } \\
\text { responsive to oral } \\
\text { administration of sumatriptan } \\
50 \text { mg assessed over one attack } \\
\text { Within-trial screening results: } \\
\text { sumatriptan non-responsive: } \\
n=220 / 347 \text {; responsive: } \\
n=124 / 347\end{array}$ & $\begin{array}{l}\text { Naratriptan } \\
\text { Oral administration of naratriptan } \\
2.5 \mathrm{mg}(n=99) \text { vs. placebo } \\
(107) \text { assessed over one attack } \\
\text { during double-blind period }\end{array}$ & $\begin{array}{l}\text { Pain relief } \mathbf{2} \text { h: naratriptan: } 25 \% \text {; } \\
\text { placebo: } 10 \% \\
\text { Pain-free } \mathbf{2} \text { h: naratriptan: } 6 \% \text {; } \\
\text { placebo: } 3 \% \\
\text { Pain relief } \mathbf{4} \text { h: naratriptan: } 41 \% \text {; } \\
\text { placebo: } 19 \% \\
\text { Pain-free } \mathbf{4} \text { h: naratriptan: } 22 \% \text {; } \\
\text { placebo: } 10 \%\end{array}$ \\
\hline $\begin{array}{l}\text { Diener, } \\
2005[21]\end{array}$ & $\begin{array}{l}\text { Randomized, blinded, } \\
\text { parallel }\end{array}$ & $\begin{array}{l}\text { Previous insufficient efficacy and/ } \\
\text { or tolerability to sumatriptan }{ }^{a} \\
\text { Within-trial screening criteria } \\
\text { for insufficient efficacy: non- } \\
\text { response to sumatriptan } 50 \mathrm{mg} \\
\text { assessed over one attack } \\
\text { Within-trial screening results: } \\
\text { sumatriptan non-responsive: } \\
\begin{array}{l}n=221 / 302 \text {; responsive } \\
n=57 / 302\end{array}\end{array}$ & $\begin{array}{l}\text { Almotriptan } \\
\text { Almotriptan } 12.5 \mathrm{mg}(n=99) \text { vs. } \\
\text { placebo }(n=99) \text { assessed over } \\
\text { one attack }\end{array}$ & $\begin{array}{l}\text { Pain relief } \mathbf{2} \mathbf{h} \text { : almotriptan } \\
\quad 12.5 \mathrm{mg} \text { : } 48 \% \text {, placebo: } 23 \% \\
\text { Pain-free } \mathbf{2} \text { h: almotriptan: } 33 \% \text {, } \\
\text { placebo: } 14 \%\end{array}$ \\
\hline
\end{tabular}


Table 1 continued

\begin{tabular}{|c|c|c|c|c|}
\hline Author, year & Study design & $\begin{array}{l}\text { Population investigated/key } \\
\text { response criteria for inclusion }\end{array}$ & Intervention investigated & Key results \\
\hline $\begin{array}{l}\text { Farkkila, } \\
2003[22]\end{array}$ & $\begin{array}{l}\text { Randomized, blinded, } \\
\text { parallel design }\end{array}$ & $\begin{array}{l}\text { Previous insufficient efficacy and/ } \\
\text { or tolerability to sumatriptan }{ }^{a}\end{array}$ & $\begin{array}{l}\text { Eletriptan } \\
\text { Eletriptan } 40 \mathrm{mg}(n=179), \\
\text { eletriptan } 80 \mathrm{mg}(n=167) \text {, vs. } \\
\text { placebo }(n=81) \text { assessed for } \\
\text { treatment of up to } 3 \text { attacks }\end{array}$ & $\begin{array}{l}\text { Pain relief } 2 \text { h: first attack: } \\
\text { eletriptan } 40 \mathrm{mg}: 59 \% \text {, } \\
\text { eletriptan } 80 \mathrm{mg} \text { : } 70 \% \text {, placebo: } \\
30 \% \\
\text { Consistency of response ( } 2 / 3 \\
\text { attacks): eletriptan } 40 \mathrm{mg}: 66 \% \text {; } \\
\text { eletriptan } 80 \mathrm{mg} \text { : } 72 \% \text {; placebo: } \\
\text { 15\% } \\
\text { Consistency of response (all } 3 \\
\text { attacks): eletriptan } 40 \mathrm{mg}: 38 \% \text {; } \\
\text { eletriptan } 80 \mathrm{mg}: 41 \% \text {; placebo: } \\
\text { 6\% } \\
\text { Pain-free } 2 \text { h: first attack: } \\
\text { eletriptan } 40 \mathrm{mg}: 35 \% \text {, } \\
\text { eletriptan } 80 \mathrm{mg}: 42 \% \text {, placebo: } \\
7 \%\end{array}$ \\
\hline $\begin{array}{l}\text { Goldstein, } \\
2006[27]\end{array}$ & Open-label & $\begin{array}{l}\text { Previous insufficient efficacy and/ } \\
\text { or tolerability to rizatriptan }\end{array}$ & $\begin{array}{l}\text { Eletriptan } \\
\text { Eletriptan } 40 \mathrm{mg}(n=121) \\
\text { assessed for first attack }\end{array}$ & $\begin{array}{l}\text { Pain relief } 2 \text { h: eletriptan } 40 \mathrm{mg} \text { : } \\
64 \%^{\mathrm{a}}(95 \% \text { CI } 55-73 \%) \\
\text { Pain-free } 2 \text { h: eletriptan } 40 \mathrm{mg}: \\
30 \% \text { ( } 95 \% \text { CI } 22-39 \% \text { ) }\end{array}$ \\
\hline $\begin{array}{l}\text { Mathew, } \\
2000[25]\end{array}$ & $\begin{array}{l}\text { Open-label, crossover } \\
\text { design }\end{array}$ & $\begin{array}{l}\text { Previous insufficient efficacy and/ } \\
\text { or tolerability to sumatriptan } \\
50 \mathrm{mg}^{\mathrm{a}}\end{array}$ & $\begin{array}{l}\text { Zomitriptan and rizatriptan } \\
\text { Group 1: zolmitriptan } 5 \mathrm{mg} \text { for } \\
\text { first } 5 \text { headaches and rizatriptan } \\
10 \mathrm{mg} \text { for next } 5 \text {; Group } 2 \text { : } \\
\text { rizatriptan } 10 \mathrm{mg} \text { followed by } \\
\text { zolmitriptan }(n=48 \text { patients; } \\
120 \text { attacks) to treat } 10 \text { attacks } \\
\text { in total }\end{array}$ & $\begin{array}{l}\text { Pain relief } \mathbf{2} \text { h (\% of attacks): } \\
\text { across } 10 \text { attacks: zolmitriptan: } \\
\text { 73\%; rizatriptan: } 81 \% \\
\text { Consistency: } 2 \text { out of } 3 \text { attacks: } \\
\text { zolmitriptan: } 81 \% \text { of attacks; } \\
\text { rizatriptan: } 80 \% \text {; } 3 \text { out of } 3 \\
\text { attacks: zolmitriptan: } 72 \% \text {; } \\
\text { rizatriptan: } 70 \% \\
\text { Pain-free } \mathbf{2} \text { h (\% of attacks): } \\
\text { across } 10 \text { attacks: zolmitriptan: } \\
\text { 45\%; rizatriptan: } 58 \%\end{array}$ \\
\hline $\begin{array}{l}\text { Newman, } \\
2008[26]\end{array}$ & Open-label, prospective & $\begin{array}{l}\text { Previous insufficient efficacy and/ } \\
\text { or tolerability to low-dose } \\
\text { sumatriptan }^{\mathrm{a}}\end{array}$ & $\begin{array}{l}\text { Sumatriptan } \\
\text { Oral administration of } \\
\text { sumatriptan } 100 \mathrm{mg} \text { (rapid } \\
\text { release) early intervention } \\
\text { (within } 30 \text { min of onset of mild } \\
\text { pain); } 4 \text { consecutive attacks } \\
\text { treated }\end{array}$ & $\begin{array}{l}\text { Pain-free } 2 \text { h: range } 53-61 \% \\
\text { across the } 4 \text { attacks }\end{array}$ \\
\hline $\begin{array}{l}\text { Landy, } \\
2004[24]\end{array}$ & $\begin{array}{l}\text { Open-label, prospective } \\
\text { in headache clinic }\end{array}$ & $\begin{array}{l}\text { Previous insufficient efficacy and/ } \\
\text { or tolerability to sumatriptan } \\
50 \mathrm{mg}^{\mathrm{a}}\end{array}$ & $\begin{array}{l}\text { Sumatriptan } \\
\text { Sumatriptan } 100 \mathrm{mg}(n=20 \\
\text { patients; } 60 \text { attacks) at the } \\
\text { earliest sign of pain while still } \\
\text { mild, in } 3 \text { subsequent attacks }\end{array}$ & $\begin{array}{l}\text { Pain-free } \mathbf{2} \text { h (\% of attacks): } 80 \% \\
\text { Sustained pain-free } 2-24 \text { h: } 75 \%\end{array}$ \\
\hline
\end{tabular}


Table 1 continued

\begin{tabular}{|c|c|c|c|c|}
\hline Author, year & Study design & $\begin{array}{l}\text { Population investigated/key } \\
\text { response criteria for inclusion }\end{array}$ & Intervention investigated & Key results \\
\hline $\begin{array}{l}\text { Mathew, } \\
2009[29]\end{array}$ & $\begin{array}{l}\text { Two identical studies: } \\
\text { randomized, double- } \\
\text { blind, placebo- } \\
\text { controlled, crossover }\end{array}$ & $\begin{array}{l}\text { Previous insufficient efficacy and/ } \\
\text { or tolerability to a short-acting } \\
\operatorname{triptan}^{\mathrm{a}}\end{array}$ & $\begin{array}{l}\text { Sumatriptan plus naproxen } \\
\text { Sumatriptan } 85 \mathrm{mg} \text { plus naproxen } \\
500 \mathrm{mg} \text { vs. placebo ( } n \text { : study } 1 \text { : } \\
\text { 144; study 2: } 139) ; 2 \text { attacks } \\
\text { treated in each trial }\end{array}$ & $\begin{array}{l}\text { Pain-free } \mathbf{2} \text { h (across attacks): } \\
\text { Study 1: } \\
\text { sumatriptan + naproxen: } 40 \% \text {; } \\
\text { placebo: } 17 \% \\
\text { Study 2: } \\
\text { sumatriptan + naproxen: } 44 \% \text {; } \\
\text { placebo: } 14 \% \\
\text { Sustained pain-free } \\
\text { 2-24 h (across attacks): } \\
\text { Study 1: } \\
\text { sumatriptan + naproxen: } 26 \% \text {; } \\
\text { placebo: } 8 \% \text {; } \\
\text { Study 2: } \\
\text { sumatriptan + naproxen: } 31 \% \text {; } \\
\text { placebo: } 8 \%\end{array}$ \\
\hline $\begin{array}{l}\text { Diamond, } \\
2007[28]\end{array}$ & $\begin{array}{l}\text { Open-label study in } \\
\text { headache clinic }\end{array}$ & $\begin{array}{l}\text { Previous insufficient efficacy and/ } \\
\text { or tolerability to any orally } \\
\text { administered triptan }\end{array}$ & $\begin{array}{l}\text { Sumatriptan } \\
6 \mathrm{mg} \text { sumatriptan }(\mathrm{SC})(n=43) \\
\text { to treat } 3 \text { attacks }\end{array}$ & $\begin{array}{l}\text { Pain relief } \mathbf{2} \text { h: first attack: } 91 \% \text {; } \\
\text { second attack: } 82 \% \text {; third } \\
\text { attack: } 72 \% \\
\text { Pain-free } \mathbf{2} \text { h: first attack: } 56 \% \text {; } \\
\text { second attack: } 49 \% \text {; third attack: } \\
51 \% \\
\text { Sustained pain free } \mathbf{2 - 2 4} \text { h: first } \\
\text { attack: } 32 \% \text {; second attack: } \\
\text { 32\%; third attack: } 35 \%\end{array}$ \\
\hline & 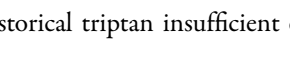 & acy and/or tolerability: subgroup & analyses with subsequent non-triptar & oral acute treatments $(n=3)$ \\
\hline $\begin{array}{l}\text { Blumenfeld, } \\
2019 \text { [32] }\end{array}$ & $\begin{array}{l}\text { Pooled subgroup analysis } \\
\text { of two randomized, } \\
\text { double-blinded, } \\
\text { studies }\end{array}$ & $\begin{array}{l}\text { Previous insufficient efficacy and/ } \\
\text { or tolerability to any triptan }\end{array}$ & $\begin{array}{l}\text { Ubrogepant } \\
\text { Ubrogepant } 50 \mathrm{mg} / 100 \mathrm{mg} \\
\text { (study 1) or ubrogepant } 25 \mathrm{mg} \text { / } \\
50 \mathrm{mg} \text { (study 2) vs. placebo } \\
\text { (1:1:1 ratio) were assessed over a } \\
\text { single attack (mITT population: } \\
\text { study 1: } n=1327 \text {; study 2: } \\
n=1355 \text { ). Baseline results: } \\
23-27 \% \text { had triptan insufficient } \\
\text { response; } 32-42 \% \text { were triptan- } \\
\text { naïv; } 35-40 \% \text { were triptan } \\
\text { responders }\end{array}$ & $\begin{array}{l}\text { Pain-free } \mathbf{2} \text { h: response rates were } \\
\text { higher for ubrogepant vs. } \\
\text { placebo across the triptan } \\
\text { subpopulations for treatment of } \\
\text { a single attack }\end{array}$ \\
\hline
\end{tabular}


Table 1 continued

\begin{tabular}{|c|c|c|c|c|}
\hline Author, year & Study design & $\begin{array}{l}\text { Population investigated/key } \\
\text { response criteria for inclusion }\end{array}$ & Intervention investigated & Key results \\
\hline $\begin{array}{l}\text { Knievel, } \\
2018[33]\end{array}$ & $\begin{array}{l}\text { Post hoc analysis of two } \\
\text { pooled randomized, } \\
\text { blinded studies }\end{array}$ & $\begin{array}{l}\text { Prior insufficient efficacy and/or } \\
\text { tolerability to any triptan }\end{array}$ & $\begin{array}{l}\text { Lasmiditan } \\
\text { Lasmiditan 100, } 200 \mathrm{mg} \text { vs. } \\
\text { placebo assessed in good, poor, } \\
\text { or non-responders }\end{array}$ & $\begin{array}{l}\text { Pain-free } \mathbf{2} \mathbf{h} \text { : benefit with } \\
\text { lasmiditan versus placebo was } \\
\text { generally unaffected by prior } \\
\text { triptan therapy response. } \\
\text { Percentages of patients } \\
\text { reporting pain freedom with } \\
\text { lasmiditan and placebo were } \\
\text { not reported in abstract }\end{array}$ \\
\hline $\begin{array}{l}\text { Ho, } \\
2011[31]\end{array}$ & $\begin{array}{l}\text { Post hoc analysis of one } \\
\text { randomized, blinded, } \\
\text { parallel design study }\end{array}$ & $\begin{array}{l}\text { Previous insufficient efficacy and/ } \\
\text { or tolerability to any triptan }\end{array}$ & $\begin{array}{l}\text { Telcagepant and zolmitriptan } \\
\text { Telcagepant } 150 \mathrm{mg} \text {, telcagepant } \\
300 \mathrm{mg} \text {, zolmitriptan } 5 \mathrm{mg} \text {, and } \\
\text { placebo (ratio 1:1:1:1) were } \\
\text { assessed in patients with good } \\
\text { historical triptan response } \\
\text { (HTR) ( } n=660) \text {, intermediate } \\
\text { HTR ( } n=248) \text {, and poor } \\
\text { HTR/no use }(n=450)\end{array}$ & $\begin{array}{l}\text { Pain relief } 2 \text { h: } \\
\text { Good HTR: zolmitriptan: } 72 \% \text {, } \\
\text { telcagepant } 300 \mathrm{mg}: 52 \% \text {; } \\
\text { telcagepant } 150 \mathrm{mg} \text { : } 48 \% \text {; } \\
\text { placebo: } 26 \% \text {; intermediate } \\
\text { HTR: zolmitriptan: } 47 \% \text {, } \\
\text { telcagepant } 300 \mathrm{mg}: 58 \% \text {; } \\
\text { telcagepant: } 58 \% \text {, placebo: } 29 \% \\
\text { Poor HTR/no use: zolmitriptan: } \\
\text { 40\%, telcagepant } 300 \text { mg: } 57 \% \text {; } \\
\text { telcagepant } 150 \text { mg: } 48 \% \text {, } \\
\text { placebo: } 31 \% \\
\text { Pain-free } 2 \text { h: } \\
\text { Good HTR: zolmitriptan: } 44 \% \text {, } \\
\text { telcagepant } 300 \text { mg: } 23 \% \text {, } \\
\text { telcagepant } 150 \text { mg: } 18 \% \\
\text { placebo: } 9 \% \\
\text { Intermediate HTR: zolmitriptan: } \\
29 \% \text {, telcagepant } 300 \text { mg: } 34 \% \text {; } \\
\text { telcagepant } 150 \text { mg: } 23 \% \text {; } \\
\text { placebo: } 8 \% \\
\text { Poor HTR/no use: zolmitriptan: } \\
\text { 14\%, telcagepant } 300 \text { mg: } 29 \% \text {; } \\
\text { telcagepant } 150 \text { mg: } 13 \% \text {, } \\
\text { placebo: } 12 \%\end{array}$ \\
\hline
\end{tabular}


Table 1 continued

\begin{tabular}{|c|c|c|c|c|}
\hline Author, year & Study design & $\begin{array}{l}\text { Population investigated/key } \\
\text { response criteria for inclusion }\end{array}$ & Intervention investigated & Key results \\
\hline \multicolumn{5}{|c|}{ Subsequent attacks: different or same triptan results $(n=2)$} \\
\hline $\begin{array}{l}\text { Tietjen, } \\
2005[34]\end{array}$ & $\begin{array}{l}\text { Randomized, crossover } \\
\text { design }\end{array}$ & $\begin{array}{l}\text { Patients with no response (self- } \\
\text { defined meaningful relief/ } \\
\text { satisfaction) to oral } \\
\text { administration of naratriptan } \\
2.5 \mathrm{mg} \text { during a migraine attack } \\
\text { were screened for enrollment; } \\
35 / 60 \text { patients had no response }\end{array}$ & $\begin{array}{l}\text { Naratriptan plus } \\
\text { prochlorperazine } \\
\text { Naratriptan } 2.5 \mathrm{mg} \text { plus } \\
\text { prochlorperazine } 25 \mathrm{mg} \text { rectal } \\
\text { suppository vs. naratriptan } \\
2.5 \mathrm{mg} \text { plus placebo were } \\
\text { assessed; subjects and } \\
\text { investigators blinded to rectal } \\
\text { suppository content; of } 15 \\
\text { patients who enrolled in the } \\
\text { study, } 14 \text { completed }\end{array}$ & $\begin{array}{l}\text { Significant decreases in headache } \\
\text { severity and disability at } 2 \mathrm{~h} \\
\text { and } 4 \mathrm{~h} \text { were observed with } \\
\text { both regimens; however, there } \\
\text { were no differences between } \\
\text { regimens }\end{array}$ \\
\hline $\begin{array}{l}\text { Landy, } \\
2014[66]\end{array}$ & $\begin{array}{l}\text { Post hoc pooled analysis } \\
\text { of } 4 \text { randomized, } \\
\text { blinded, studies }\end{array}$ & $\begin{array}{l}\text { Insufficient efficacy with } \\
\text { eletriptan } 40 \mathrm{mg} \text { in the first } \\
\text { attack (intervention results } \\
\text { reported on the basis of } \\
\text { patients who did not achieve } \\
\text { pain relief at } 2 \mathrm{~h} \text { with eletriptan } \\
40 \mathrm{mg} \text { in attack } 1 \text { ) }\end{array}$ & $\begin{array}{l}\text { Eletriptan } \\
\text { Eletriptan } 40 \text { mg vs. placebo was } \\
\text { assessed over } 3 \text { attacks. First } \\
\text { attack screening results: no pain } \\
\text { relief at } 2 \text { h: eletriptan } 40 \mathrm{mg} \text { : } \\
\text { 297/1299 patients, placebo } \\
\text { 375/1299 patients. Patients } \\
\text { with no } 2 \text { h pain relief with } \\
\text { eletriptan in first attack and who } \\
\text { treated all three attacks received } \\
\text { eletriptan } 40 \mathrm{mg}(n=170) \text { or } \\
\text { placebo }(n=193) \text { in the } 2 \text { nd } \\
\text { and } 3 \text { rd attacks }\end{array}$ & $\begin{array}{l}\text { Pain relief } 2 \text { h: attack 2: } \\
\text { eletriptan 49\%; placebo } 20 \% \text {; } \\
\text { attack 3: eletriptan: } 37 \% \text {; } \\
\text { placebo } 16 \% \\
\text { Pain free } 2 \text { h: attack 2: eletriptan } \\
\text { 17\%; placebo } 4 \% \text {; attack 3: } \\
\text { eletriptan 19\%; placebo 3\% }\end{array}$ \\
\hline \multicolumn{5}{|c|}{ Same attack: second dose with same treatment $(n=2)$} \\
\hline $\begin{array}{l}\text { Scott, } \\
1996[64]\end{array}$ & $\begin{array}{l}\text { Randomized, blinded, } \\
\text { parallel design } \\
\text { (conducted in general } \\
\text { practice centers) }\end{array}$ & $\begin{array}{l}\text { Insufficient efficacy to the first } \\
\text { dose of sumatriptan } 100 \mathrm{mg} \\
\text { (no pain relief at } 4 \mathrm{~h} \text { ) }\end{array}$ & $\begin{array}{l}\text { Sumatriptan } \\
\text { Sumatriptan } 100 \mathrm{mg} \text { (second } \\
\text { dose) vs. placebo assessed in } \\
\text { patients who did not respond to } \\
\text { first dose } \\
\text { First-dose screening results: lack of } \\
\text { pain relief at } 4 \mathrm{~h} \text { with } \\
\text { sumatriptan } 100 \mathrm{mg} \text { in } 30 \% \text { of } \\
1349 \text { patients }\end{array}$ & $\begin{array}{l}\text { Pain relief } 4 \text { h: after second dose } \\
\text { (range over } 3 \text { treated attacks): } \\
\text { sumatriptan } 100 \mathrm{mg} \text { : } 51-63 \% \text {; } \\
\text { placebo: } 51-61 \%\end{array}$ \\
\hline
\end{tabular}


Table 1 continued

\begin{tabular}{|c|c|c|c|c|}
\hline Author, year & Study design & $\begin{array}{l}\text { Population investigated/key } \\
\text { response criteria for inclusion }\end{array}$ & Intervention investigated & Key results \\
\hline $\begin{array}{l}\text { Spierings, } \\
2009[67]\end{array}$ & $\begin{array}{l}\text { Randomized, double- } \\
\text { blinded }\end{array}$ & $\begin{array}{l}\text { Insufficient efficacy to the first } \\
\text { dose of frovatriptan } 2.5 \mathrm{mg}\end{array}$ & $\begin{array}{l}\text { Frovatriptan } \\
\text { Frovatriptan } 2.5 \mathrm{mg} \text { for first } \\
\text { attack; frovatriptan } 2.5 \mathrm{mg} \text { vs. } \\
\text { placebo for second attack } \\
(n=547 \text { enrolled patients of } \\
\text { which } 486 \text { treated moderate or } \\
\text { severe headache) }\end{array}$ & $\begin{array}{l}\text { In first attack, } 63 \% \text { of } 486 \text { patients } \\
\text { with moderate or severe } \\
\text { headache had no pain relief at } \\
2 \mathrm{~h} \text { after the first dose. Of } 486 \\
\text { patients, } 173 \text { ( } 36 \% \text { ) did not } \\
\text { take a second dose of study } \\
\text { medication at } 2 \text { h for non- } \\
\text { response (rapid responders). } \\
\text { Among rapid responders, } 84 \% \\
\text { and } 98 \% \text { reported mild or no } \\
\text { pain at } 2 \text { h and } 4 \text { h, } \\
\text { respectively. Among rapid } \\
\text { responders, } 24 \text {-h recurrence rate } \\
\text { was } 6 \% \\
\text { Second attack results not provided }\end{array}$ \\
\hline \multicolumn{5}{|c|}{ Predictors of insufficient efficacy within a single attack $(n=2)$} \\
\hline $\begin{array}{l}\text { Cady, } \\
2007[35]\end{array}$ & $\begin{array}{l}\text { Two identical } \\
\text { randomized, blinded } \\
\text { studies } \\
\text { Aim: assessment of } \\
\text { patient-reported } \\
\text { symptoms of cutaneous } \\
\text { sensitivity (SCS) as a } \\
\text { negative predictor of } \\
\text { response }\end{array}$ & $\begin{array}{l}\text { Patients with migraine with/ } \\
\text { without aura with history of } \\
\text { attacks that were typically mild } \\
\text { at onset }\end{array}$ & $\begin{array}{l}\text { Rizatriptan } \\
\text { Rizatriptan } 10 \text { mg or placebo with } \\
\text { early treatment (within } 1 \mathrm{~h} \\
\text { while pain still mild) was } \\
\text { assessed over one attack } \\
\text { Symptoms of SCS were present } \\
\text { pre treatment in } 22-29 \% \text { of } \\
\text { patients across the two studies } \\
\text { Patient numbers were rizatriptan: } \\
n=353 \text { (study 1) and } 331 \\
\text { (study 2); placebo: } n=177 \\
\text { (study 1) and } 169 \text { (study } 2 \text { ) }\end{array}$ & $\begin{array}{l}\text { Pain-free } \mathbf{2} \text { h: } \\
\text { Baseline symptoms of SCS: } \\
\text { rizatriptan 55-58\%; placebo } \\
24-35 \% \\
\text { No baseline symptoms of SCS: } \\
\text { rizatriptan 58-59\%; placebo } \\
29-33 \%\end{array}$ \\
\hline $\begin{array}{l}\text { Diener, } \\
2008[45]\end{array}$ & $\begin{array}{l}\text { Pooled analysis of } 10 \\
\text { randomized, blinded, } \\
\text { parallel design studies }\end{array}$ & $\begin{array}{l}\text { Patients with migraine with/ } \\
\text { without aura }\end{array}$ & $\begin{array}{l}\text { Eletriptan } \\
\text { Negative predictors of response } \\
\text { with eletriptan } 20 \mathrm{mg} \text {, eletriptan } \\
40 \mathrm{mg} \text {, eletriptan } 80 \mathrm{mg} \text {, or } \\
\text { sumatriptan } 100 \mathrm{mg} \text { vs. placebo } \\
\text { were assessed ( } n=8473 \\
\text { patients for first-attack data) }\end{array}$ & $\begin{array}{l}\text { Pain free } 2 \text { h: eletriptan } 20 \mathrm{mg} \text { : } \\
\quad \sim 15 \% \text {; eletriptan } 40 \mathrm{mg}: \\
\sim 30 \% \text {; eletriptan } 80 \mathrm{mg}: \\
\sim 35 \% \text {, sumatriptan } 100 \mathrm{mg}: \\
\sim 25 \% \text {; placebo: } \sim 5 \% \\
\text { Multivariate regression analyses } \\
\text { identified severe headache pain, } \\
\text { presence of photophobia/ } \\
\text { phonophobia, and presence of } \\
\text { nausea as significant baseline } \\
\text { predictors of failure to achieve } \\
\text { 2-h pain-free response. Time of } \\
\text { dosing following headache onset } \\
\text { did not show influence }\end{array}$ \\
\hline
\end{tabular}


Table 1 continued

\begin{tabular}{|c|c|c|c|c|}
\hline Author, year & Study design & $\begin{array}{l}\text { Population investigated/key } \\
\text { response criteria for inclusion }\end{array}$ & Intervention investigated & Key results \\
\hline \multicolumn{5}{|c|}{ Repeated attacks insufficient efficacy $(n=1)$} \\
\hline $\begin{array}{l}\text { Miljkovic, } \\
2018[70]\end{array}$ & $\begin{array}{l}\text { Randomized, blinded, } \\
\text { parallel design }\end{array}$ & $\begin{array}{l}\text { Patients with migraine without } \\
\text { aura }\end{array}$ & $\begin{array}{l}\text { Ergot-based formulation vs. } \\
\text { sumatriptan } \\
\text { Ergot-based formulation } \\
\quad\left(\text { Nomigren }{ }^{\circledR}\right) \text { vs. sumatriptan } \\
\quad(\text { dose unspecified) was assessed } \\
(n=201 \text { patients) }\end{array}$ & $\begin{array}{l}\text { Pain-free } 2 \mathbf{h} \text { : Nomigren }{ }^{\circledR}: 51 \% \text {; } \\
\text { sumatriptan: } 34 \% \text {; } \\
\text { Pain-free } 2 \text { h: repeat attacks only: } \\
\text { Nomigren }{ }^{\circledR}: 51 \% \text {, sumatriptan: } \\
24 \%\end{array}$ \\
\hline \multicolumn{5}{|c|}{ Menstrual migraine $(n=2)$} \\
\hline $\begin{array}{l}\text { Brandes, } \\
2009[63]\end{array}$ & $\begin{array}{l}\text { Randomized, blinded, } \\
\text { parallel design }\end{array}$ & $\begin{array}{l}\text { Patients with difficult-to-treat } \\
\text { menstrual migraine and } \\
\text { historical insufficient efficacy } \\
\text { and/or tolerability to any } \\
\text { triptan }^{a}\end{array}$ & $\begin{array}{l}\text { Frovatriptan } \\
\text { Frovatriptan } 2.5 \mathrm{mg} \text { (loading } \\
\text { dose: } 5 \mathrm{mg} \text { ) vs. placebo was } \\
\text { assessed for short-term } \\
\text { prevention of migraine. Two } \\
\text { dosing regimens (qd or bid) } \\
\text { were studied. A 6-day regimen } \\
\text { (starting } 2 \text { days prior to } \\
\text { anticipated migraine) was used } \\
(n=410)\end{array}$ & $\begin{array}{l}\text { Headache-free peri-menstrual } \\
\text { periods (mean number of per } \\
\text { patient): frovatriptan qd: } 0.69 \text {; } \\
\text { frovatriptan bid: } 0.92 \text {; placebo: } \\
0.42 \\
\text { Severity and incidence of difficult- } \\
\text { to-treat menstrual migraine were } \\
\text { significantly reduced in both } \\
\text { frovatriptan arms }\end{array}$ \\
\hline $\begin{array}{l}\text { Cady, } \\
2014[65]\end{array}$ & $\begin{array}{l}\text { Randomized, blinded, } \\
\text { parallel design }\end{array}$ & $\begin{array}{l}\text { Patients with menstrual migraine } \\
\text { Single menstrual migraine episode }\end{array}$ & $\begin{array}{l}\text { Sumatriptan plus naproxen } \\
\text { Sumatriptan } 85 \mathrm{mg}+\text { naproxen } \\
500 \mathrm{mg} \text { (Treximet) }(n=21) \text { vs. } \\
\text { placebo ( } n=20) \text { were to be } \\
\text { taken at onset of menstrual } \\
\text { migraine episode }\end{array}$ & $\begin{array}{l}\text { Migraine recurrence within } 24 \mathrm{~h} \\
\text { post treatment occurred in } \\
2 / 14 \text { patients treated with } \\
\text { Treximet and } 0 / 11 \text { placebo- } \\
\text { treated patients } \\
\text { Mean time to pain-free was: } \\
\text { Treximet: } 3.9 \mathrm{~h}(n=14) ; \\
\text { placebo: } 7.64 \mathrm{~h}(n=10)\end{array}$ \\
\hline
\end{tabular}

bid twice daily, $h$ hour, $q d$ once daily

${ }^{\text {a }}$ Criteria to define historical insufficient efficacy and/or tolerability for each of the 10 studies is described in Table 3 below

those three studies, $21-36 \%$ of patients who reported prior insufficient efficacy to sumatriptan experienced pain relief at $2 \mathrm{~h}$ with sumatriptan during the trial. Among the patients with confirmed insufficient efficacy and/or tolerability, for the outcome of pain freedom at $2 \mathrm{~h}, 6 \%$ subsequently responded to naratriptan $2.5 \mathrm{mg}, 33 \%$ to almotriptan $12.5 \mathrm{mg}$, and $22 \%$ to rizatriptan $10 \mathrm{mg}$ ODT (orally disintegrating tablets). For the outcome of pain relief at $2 \mathrm{~h}$, $25 \%$ subsequently responded to naratriptan $2.5 \mathrm{mg}, 48 \%$ to almotriptan $12.5 \mathrm{mg}$, and $51 \%$ to rizatriptan $10 \mathrm{mg}$ (ODT; over three attacks). In all three studies, patients were instructed to treat the migraine attack when pain was moderate or severe.

Three of the ten studies investigated responses to a different orally administered triptan (monotherapy) without a run-in validation phase. Two were early intervention studies and are discussed in the respective sections $[25,27]$. The other, in which patients were instructed to treat while pain was moderate or severe, was conducted in patients who had previously discontinued orally administered sumatriptan [30]. In that study, 35-42\% experienced pain freedom at $2 \mathrm{~h}$ and $59-70 \%$ of patients subsequently treated with eletriptan $40 \mathrm{mg}$ or $80 \mathrm{mg}$ experienced pain relief at $2 \mathrm{~h}$. 
Table 2 Study characteristics and key results of observational studies

\begin{tabular}{|c|c|c|c|c|}
\hline Author, year & Study design & $\begin{array}{l}\text { Patient } \\
\text { population; } \\
\text { characteristics } \\
\text { investigated }\end{array}$ & $\begin{array}{l}\text { Study description or } \\
\text { intervention }\end{array}$ & Results (key relevant findings) \\
\hline \multicolumn{5}{|c|}{ Characteristics investigated in triptan insufficient efficacy and/or tolerability } \\
\hline $\begin{array}{l}\text { Sheftell, } \\
2010[42]\end{array}$ & $\begin{array}{l}\text { 6-month } \\
\text { observational } \\
\text { study using } \\
\text { data recorded } \\
\text { in e-diaries }\end{array}$ & $\begin{array}{l}\text { Patients with } \\
\text { migraine who } \\
\text { were prescribed } \\
\text { any triptan }\end{array}$ & $\begin{array}{l}\text { Any triptan } \\
\text { Headache return with any triptan } \\
\text { including sumatriptan, } \\
\text { rizatriptan, eletriptan, } \\
\text { zolmitriptan, almotriptan, } \\
\text { sumatriptan injection, triptan } \\
\text { nasal spray ( } n=359 \text { patients } \\
\text { who treated } 2168 \text { attacks) }\end{array}$ & $\begin{array}{l}\text { Pain freedom or pain relief at } \\
\mathbf{2} \text { h: } 66 \% \text { of attacks } \\
\text { Headache returned by } 48 \text { h: } \\
19 \% \text { of attacks, across } 320 \\
\text { patients } \\
\text { Factors associated with } \\
\text { increased likelihood of } \\
\text { headache return: more severe } \\
\text { baseline headache, short } \\
\text { duration since diagnosis of } \\
\text { migraine, female gender }\end{array}$ \\
\hline $\begin{array}{c}\text { Terrazzino, } \\
2010[43]\end{array}$ & Cohort study & $\begin{array}{l}\text { Patients with } \\
\text { migraine with/ } \\
\text { without aura }\end{array}$ & $\begin{array}{l}\text { Any triptan } \\
\text { Specific genetic markers } \\
\text { predictive of consistency in } \\
\text { headache response to any } \\
\text { triptan including eletriptan, } \\
\text { rizatriptan, almotriptan, } \\
\text { frovatriptan, sumatriptan, and } \\
\text { zolmitriptan were assessed }\end{array}$ & $\begin{array}{l}\text { Consistent response to triptans } \\
\text { was observed in } 67 \% \text { of } \\
\text { patients with migraine ( } 87 / \\
\text { 130); } 33 \% \text { (43/130) of } \\
\text { patients did not consistently } \\
\text { respond. Consistent } \\
\text { responders to each triptan } \\
\text { (n): eletriptan: } 33 \text {; rizatriptan: } \\
\text { 29; sumatriptan: 20; } \\
\text { frovatriptan: } 18 \text {; almotriptan: } \\
\text { 21; zolmitriptan: } 9 \\
\text { Patients who had undergone } \\
\text { preventive treatment versus } \\
\text { who had not appeared to, } \\
\text { responded more consistently } \\
\text { to triptan therapy } \\
\text { Results support role of STin2 } \\
\text { VNTR polymorphism of } \\
\text { serotonin transporter gene in } \\
\text { conferring higher risk of } \\
\text { inconsistent response to } \\
\text { triptans }\end{array}$ \\
\hline
\end{tabular}


Table 2 continued

\begin{tabular}{|c|c|c|c|c|}
\hline Author, year & Study design & $\begin{array}{l}\text { Patient } \\
\text { population; } \\
\text { characteristics } \\
\text { investigated }\end{array}$ & $\begin{array}{l}\text { Study description or } \\
\text { intervention }\end{array}$ & Results (key relevant findings) \\
\hline $\begin{array}{l}\text { Munjal, } \\
2016[36]\end{array}$ & Cohort study & $\begin{array}{l}\text { Patients with } \\
\text { episodic } \\
\text { migraine }\end{array}$ & $\begin{array}{l}\text { Any triptan } \\
\text { Impact of allodynia on triptan } \\
\text { (any) response }\end{array}$ & $\begin{array}{l}\text { Pain-free } 2 \mathrm{~h} \text { : overall: } 44 \% \\
(n=3621) \\
\text { No pain-free } 2 \mathrm{~h} \text { : triptan users } \\
\text { with allodynia: } 50 \% \text {; triptan } \\
\text { users without allodynia: } 36 \% \text {; } \\
\text { non-triptan use with } \\
\text { allodynia: } 65 \% \text {; non-triptan } \\
\text { use without allodynia: } 51 \% \\
\text { Inadequate response to } \\
\text { medication more likely in the } \\
\text { presence of allodynia among } \\
\text { triptan and non-triptan users }\end{array}$ \\
\hline $\begin{array}{l}\text { Silberstein, } \\
2019[37]\end{array}$ & $\begin{array}{l}\text { Case-control } \\
\text { study }\end{array}$ & $\begin{array}{l}\text { Patients with } \\
\text { migraine }\end{array}$ & $\begin{array}{l}\text { Any triptan } \\
\text { Opioid use, rebound headache, } \\
\text { and healthcare resource } \\
\text { utilization with any triptan } \\
\text { were analyzed using data from } \\
\text { electronic medical records, and } \\
\text { patient and physician surveys }\end{array}$ & $\begin{array}{l}\text { Triptan insufficient responders } \\
\text { were } 3 \text { times more likely than } \\
\text { responders to suffer from } \\
\text { rebound headaches, } 3 \text { times } \\
\text { more likely to be admitted to } \\
\text { a hospital in the past year, } 13 \\
\text { times more likely to receive an } \\
\text { opioid, and had } 37 \% \text { more } \\
\text { visits to a healthcare } \\
\text { professional in the past year }\end{array}$ \\
\hline $\begin{array}{l}\text { Peng, } \\
2016[40]\end{array}$ & $\begin{array}{l}\text { Cohort study in } \\
\text { headache } \\
\text { clinic }\end{array}$ & $\begin{array}{l}\text { Patients with } \\
\text { migraine using/ } \\
\text { recently } \\
\text { prescribed } \\
\text { sumatriptan }\end{array}$ & $\begin{array}{l}\text { Sumatriptan } \\
\text { Patients were asked for } \\
\text { effectiveness and AE of } \\
\text { sumatriptan } 50 \mathrm{mg} \text { for their } \\
\text { migraine attacks }(n=1024 \\
\text { patients); demographics and } \\
\text { comorbidity characteristics } \\
\text { assessed }\end{array}$ & $\begin{array}{l}\text { Overall effectiveness (definition } \\
\text { unspecified) rate of treatment } \\
\text { with one tablet sumatriptan } \\
50 \mathrm{mg} \text { : } 61.3 \% \\
\text { Responders to sumatriptan, } \\
\text { compared to non-responders, } \\
\text { were older (mean } 40.6 \text { vs. } \\
37.2 \text { years), had milder } \\
\text { headache intensity (mean } 6.4 \\
\text { vs. } 6.7 \text { on } 0-10 \text { scale), had } \\
\text { lower scores in Beck } \\
\text { Depression Inventory (mean } \\
10.6 \text { vs. } 12.1 \text { ), and have regular } \\
\text { coffee intake }\end{array}$ \\
\hline
\end{tabular}


Table 2 continued

\begin{tabular}{|c|c|c|c|c|}
\hline Author, year & Study design & $\begin{array}{l}\text { Patient } \\
\text { population; } \\
\text { characteristics } \\
\text { investigated }\end{array}$ & $\begin{array}{l}\text { Study description or } \\
\text { intervention }\end{array}$ & Results (key relevant findings) \\
\hline $\begin{array}{l}\text { Wang, } \\
2017 \text { [44] }\end{array}$ & $\begin{array}{l}\text { Cohort study in } \\
\text { headache } \\
\text { clinic }\end{array}$ & $\begin{array}{l}\text { Patients with } \\
\text { migraine } \\
\text { prescribed with } \\
\text { sumatriptan }\end{array}$ & $\begin{array}{l}\text { Sumatriptan } \\
\text { Sumatriptan } 50 \mathrm{mg} \text { tablet } \\
\quad(n=1499 \text { enrolled patients })\end{array}$ & $\begin{array}{l}\text { Pain relief } \mathbf{2} \mathbf{h} \text { (in at least } 2 \text { out } \\
\text { of } 3 \text { ) attacks: } 69 \% \\
\text { Regular coffee consumption } \\
\text { positively associated with } \\
\text { effectiveness of sumatriptan. } \\
\text { Compared to non-responders, } \\
\text { responders had lower } \\
\text { psychiatric measures (Beck } \\
\text { Depression Inventory: mean } \\
10.5 \text { vs. } 11.8 \text { ) and lower } \\
\text { baseline headache frequency } \\
\text { (mean } 9.8 \text { vs. } 11.6 \text { days per } \\
\text { month) }\end{array}$ \\
\hline $\begin{array}{l}\text { Patrick, } \\
2000 \text { [39] }\end{array}$ & $\begin{array}{l}\text { Long-term } \\
\text { (1 year) } \\
\text { observational } \\
\text { cohort study }\end{array}$ & $\begin{array}{l}\text { Patients with } \\
\text { migraine who } \\
\text { had participated } \\
\text { in zolmitriptan } \\
\text { trials }\end{array}$ & $\begin{array}{l}\text { Zolmitriptan } \\
\text { Quality of life associated with } \\
\text { oral administration of } \\
\text { zolmitriptan } 5 \mathrm{mg}(n=1383)\end{array}$ & $\begin{array}{l}\text { A significantly greater } \\
\text { improvement in MSQOL } \\
\text { score was observed in patients } \\
\text { who had pain relief at } 2 \mathrm{~h} \text { and } \\
\text { pain freedom at } 2 \mathrm{~h}, \\
\text { compared with non- } \\
\text { responders } \\
\text { Post treatment, responders had } \\
\text { MSQOL scores approximately } \\
5 \text { points higher than those of } \\
\text { non-responders } \\
\text { Non-responders had lower } \\
\text { baseline MSQOL scores (by } \\
\text { approximately } 4 \text { to } 7 \text { points) } \\
\text { than responders }\end{array}$ \\
\hline
\end{tabular}


Table 2 continued

\begin{tabular}{|c|c|c|c|c|}
\hline Author, year & Study design & $\begin{array}{l}\text { Patient } \\
\text { population; } \\
\text { characteristics } \\
\text { investigated }\end{array}$ & $\begin{array}{l}\text { Study description or } \\
\text { intervention }\end{array}$ & Results (key relevant findings) \\
\hline $\begin{array}{l}\text { Sarchielli, } \\
2006[38]\end{array}$ & $\begin{array}{l}\text { Cohort study } \\
\text { across } 3 \\
\text { headache } \\
\text { centers }\end{array}$ & $\begin{array}{l}\text { Triptan-naïve } \\
\text { patients with } \\
\text { migraine } \\
\text { without aura }\end{array}$ & $\begin{array}{l}\text { Rizatriptan } \\
\text { Clinical and biochemical } \\
\text { correlates associated with } \\
\text { rizatriptan responders and non- } \\
\text { responders }(n=20,10 \text { each for } \\
\text { responders and non- } \\
\text { responders). Response was } \\
\text { defined as achieving pain relief } \\
\text { at } 2 \mathrm{~h} \text { (without } 48 \mathrm{~h} \\
\text { recurrence) to rizatriptan } 10 \mathrm{mg} \\
\text { in at least } 3 \text { consecutive attacks }\end{array}$ & $\begin{array}{l}\text { CGRP and NKA levels } \\
\text { measured at baseline were } \\
\text { significantly higher in those } \\
\text { with baseline sufficient } \\
\text { efficacy vs. those with } \\
\text { insufficient efficacy } \\
1 \text { h after rizatriptan } \\
\text { administration, a decrease in } \\
\text { CGRP and NKA levels was } \\
\text { evident in those with sufficient } \\
\text { efficacy, and this corresponded } \\
\text { to significant pain relief and } \\
\text { alleviation of accompanying } \\
\text { symptoms. Those with } \\
\text { insufficient efficacy had less } \\
\text { significant variations in CGRP } \\
\text { and NKA levels at all time } \\
\text { points } \\
\text { Insufficient efficacy appeared to } \\
\text { be correlated with a lesser } \\
\text { degree of trigeminal activation, } \\
\text { lower variations of trigeminal } \\
\text { neuropeptides after triptan } \\
\text { administration, and no } \\
\text { evidence of parasympathetic } \\
\text { activation at baseline }\end{array}$ \\
\hline
\end{tabular}


Table 2 continued

\begin{tabular}{|c|c|c|c|c|}
\hline Author, year & Study design & $\begin{array}{l}\text { Patient } \\
\text { population; } \\
\text { characteristics } \\
\text { investigated }\end{array}$ & $\begin{array}{l}\text { Study description or } \\
\text { intervention }\end{array}$ & Results (key relevant findings) \\
\hline $\begin{array}{l}\text { Seo, } \\
2016[41]\end{array}$ & $\begin{array}{l}\text { Observational } \\
\text { case-control } \\
\text { study in } \\
\text { headache } \\
\text { clinic }\end{array}$ & $\begin{array}{l}\text { Triptan-naïve } \\
\text { patients with } \\
\text { migraine }\end{array}$ & $\begin{array}{l}\text { Frovatriptan } \\
\text { Patients were instructed to take } \\
\text { frovatriptan } 2.5 \mathrm{mg} \text { as early as } \\
\text { possible after migraine attack. } \\
\text { Demographic, clinical, and } \\
\text { psychiatric variables were } \\
\text { investigated ( } n=128 \text { patients) }\end{array}$ & $\begin{array}{l}\text { No pain relief } \mathbf{4} \mathbf{h} \text { in at least } 1 \\
\text { out of } 2 \text { consecutive attacks in } \\
22 \% \text { of patients. In patients } \\
\text { with major depressive } \\
\text { disorder (MDD), } 50 \% \text { had no } \\
\text { pain relief ( } 4 \text { h); MDD was } \\
\text { identified as a risk factor } \\
\text { Pain relief } 4 \mathbf{h} \text { in at least } 1 \text { out } \\
\text { of } 2 \text { consecutive attacks in } \\
78 \% \text { of patients; } 92 \% \text { of } \\
\text { responder patients, achieved } \\
\text { response in first attack and } \\
\text { responded in second attack as } \\
\text { well }\end{array}$ \\
\hline \multicolumn{5}{|c|}{ Specific populations } \\
\hline $\begin{array}{l}\text { al Deeb, } \\
1997 \text { [69] }\end{array}$ & $\begin{array}{l}\text { Prospective } \\
\text { cohort study } \\
\text { in } 2 \text { hospitals }\end{array}$ & $\begin{array}{l}\text { Patients with } \\
\text { migraine in } \\
\text { Saudi Arabia }\end{array}$ & $\begin{array}{l}\text { Sumatriptan } \\
\text { Orally administered sumatriptan } \\
100 \text { mg to be taken as soon as } \\
\text { possible and maximally within } \\
2 \mathrm{~h} \text { after each of two attacks } \\
\text { ( } n=63 \text { patients). Better } \\
\text { response of two attacks } \\
\text { reported. Neurologist assessed } \\
\text { efficacy on a 4-point scale on } \\
\text { the basis pain diminution along } \\
\text { a pain severity scale (severe: } 3 \text {; } \\
\text { moderate: } 2 \text {; mild: } 1 \text {; none: } 0)\end{array}$ & $\begin{array}{l}\text { 3-point drop } 4 \mathbf{h} \text { : } 33 \% \\
\text { 2-point drop } \mathbf{4} \mathbf{h} \text { (better of } 2 \\
\text { treated attacks): } 33 \% \\
\text { 1-point drop } \mathbf{4} \mathbf{h} \text { (better of } 2 \\
\text { treated attacks): } 14 \% \\
\text { No drop } 4 \mathbf{h} \text { in either attack: } \\
\text { 19\% }\end{array}$ \\
\hline $\begin{array}{l}\text { Linder, } \\
1996[71]\end{array}$ & Cohort study & $\begin{array}{l}\text { Pediatric patients } \\
\text { with migraine }\end{array}$ & $\begin{array}{l}\text { Sumatriptan }(\mathbf{S C}) \\
\text { Subcutaneously administered } \\
\text { sumatriptan } 0.06 \mathrm{mg} / \mathrm{kg} \\
(n=50 \text { children })\end{array}$ & $\begin{array}{l}\text { Pain relief reported in } 78 \% \text { of } \\
\text { patients; } 22 \% \text { had no or } \\
\text { suboptimal response } \\
\text { Migraine with episodic tension- } \\
\text { type headaches/chronic } \\
\text { tension-type headaches was } \\
\text { much more frequent in the } \\
\text { female patients and, in general, } \\
\text { was more difficult to treat }\end{array}$ \\
\hline
\end{tabular}


Table 2 continued

\begin{tabular}{lllll}
\hline Author, year Study design & $\begin{array}{l}\text { Patient } \\
\text { population; } \\
\text { characteristics } \\
\text { investigated }\end{array}$ & $\begin{array}{l}\text { Study description or } \\
\text { intervention }\end{array}$ & Results (key relevant findings) \\
\hline
\end{tabular}

Switching or discontinuation of triptans

\begin{tabular}{|c|c|c|c|c|}
\hline $\begin{array}{l}\text { Sheftell, } \\
2004[68]\end{array}$ & $\begin{array}{l}\text { Retrospective } \\
\text { cohort study }\end{array}$ & $\begin{array}{l}\text { Patients with } \\
\text { migraine with } \\
\text { current use of } \\
\text { triptans }\end{array}$ & $\begin{array}{l}\text { Any triptan } \\
\text { Any triptan including } \\
\text { sumatriptan (subcutaneous, } \\
\text { nasal, oral), zolmitriptan, } \\
\text { rizatriptan, naratriptan and, } \\
\text { almotriptan was allowed }\end{array}$ & $\begin{array}{l}\text { Incomplete or no relief as } \\
\text { reason for switching a triptan, } \\
n \text { (\%): sumatriptan } 25 \mathrm{mg:} 89 \\
\text { (70.1); sumatriptan } 50 \mathrm{mg:} \\
105 \text { (33.3); sumatriptan } \\
100 \text { mg: } 62 \text { (27.2); } \\
\text { sumatriptan nasal spray: } 17 \\
\text { (37.7); sumatriptan SC: } 10 \\
\text { (12.2); zolmitriptan: } 69 \\
\text { (28.9); rizatriptan: } 52 \text { (26.7); } \\
\text { naratriptan: } 54 \text { (39.4) } \\
\text { "Incomplete or no relief were } \\
\text { most common most frequent } \\
\text { switches in } 6 \text { out of the } 8 \\
\text { assessed formulations. Side } \\
\text { effects were the most frequent } \\
\text { cause leading to switches in } \\
\text { the remaining two } \\
\text { (sumatriptan } 100 \text { mg and } \\
\text { sumatriptan SC)" } \\
\text { "Patients using sumatriptan } \\
25 \text { mg were more likely to } \\
\text { report that the other triptans } \\
\text { they used to be better, while } \\
\text { patients using sumatriptan SC } \\
\text { were less likely to do so. For all } \\
\text { the other } \\
\text { triptan/formulations, patients } \\
\text { were equally likely, from a } \\
\text { statistical perspective, to report } \\
\text { that other triptan they had } \\
\text { used was better. More patients } \\
\text { who used sumatriptan } 50 \text { or } \\
100 \text { mg as the initial triptan } \\
\text { were likely to switch back to } \\
\text { it" }\end{array}$ \\
\hline
\end{tabular}


Table 2 continued

\begin{tabular}{|c|c|c|c|c|}
\hline Author, year & Study design & $\begin{array}{l}\text { Patient } \\
\text { population; } \\
\text { characteristics } \\
\text { investigated }\end{array}$ & $\begin{array}{l}\text { Study description or } \\
\text { intervention }\end{array}$ & Results (key relevant findings) \\
\hline $\begin{array}{l}\text { Alam, } \\
2018 \text { [72] }\end{array}$ & $\begin{array}{l}\text { Cohort study: } \\
\text { Web-based } \\
\text { survey }\end{array}$ & $\begin{array}{l}\text { Respondents } \\
\text { using acute } \\
\text { prescription } \\
\text { migraine } \\
\text { medications }\end{array}$ & $\begin{array}{l}\text { Any triptan } \\
\text { Overall triptan use and } \\
\text { discontinuation with } \\
\text { oral/nasal/injectable triptan }\end{array}$ & $\begin{array}{l}\text { Discontinuation rates were } \\
\text { highest for } \\
\text { injectables }(81.5 \%) \text {, nasal } \\
\text { sprays }(66.5 \%) \text {, and oral } \\
\text { medications }(55.2 \%)\end{array}$ \\
\hline & & & & $\begin{array}{l}\text { Lack of efficacy and side effects } \\
\text { were the main reasons for } \\
\text { discontinuation }\end{array}$ \\
\hline
\end{tabular}

$b$ hour, $S C$ subcutaneous

Three studies investigating subsequent responses to a higher dose and/or alternative formulation of sumatriptan $[24,26,28]$, and one publication describing subsequent response to combination treatment, are summarized in respective sections [29].

In addition to the ten studies described earlier, three subgroup analyses identified and assessed outcomes to subsequent acute treatments in patients with prior triptan insufficient efficacy and/or tolerability from a broader group of patients within RCTs; subsequent treatments investigated were telcagepant, zolmitriptan, ubrogepant, and lasmiditan [31-33]. In the analysis assessing outcomes with telcagepant and zolmitriptan, percentage of patients with historical poor triptan response or no triptan use experiencing pain freedom at $2 \mathrm{~h}$ was $13 \%$ with telcagepant $150 \mathrm{mg}, 14 \%$ with zolmitrip$\tan 5 \mathrm{mg}, 29 \%$ with telcagepant $300 \mathrm{mg}$, and $12 \%$ with placebo [31]. The therapeutic benefit of lasmiditan versus placebo for the outcome of pain-free response at $2 \mathrm{~h}$ was generally unaffected by prior response to triptan therapy [33]. Response rates for 2-h pain freedom were higher for ubrogepant versus placebo, and the magnitude of benefit was similar across triptan-responder, triptan-insufficient responder, and triptan-naïve patient categories [32].

\section{Early Intervention with a Different Triptan}

In two studies, in patients with reported prior triptan insufficient efficacy and/or tolerability, efficacy to a different orally administered triptan as monotherapy was assessed when treatment was administered early (e.g., within $1 \mathrm{~h}$ of attack onset) and/or while headache severity was still mild $[25,27]$. Across these studies, pain freedom at $2 \mathrm{~h}$ was experienced by $30 \%$ of patients who took eletriptan $40 \mathrm{mg}$, and in $45 \%$ (zolmitriptan $5 \mathrm{mg}$ ) to $58 \%$ (rizatriptan $10 \mathrm{mg}$ ) of attacks. Pain relief at $2 \mathrm{~h}$ was experienced by $64 \%$ of patients who took eletriptan $40 \mathrm{mg}$, and in $73 \%$ (zolmitriptan $5 \mathrm{mg}$ ) to $81 \%$ (rizatriptan $10 \mathrm{mg}$ ) of attacks.

\section{Higher Dose and/or Alternative Triptan Formulation}

In four studies conducted in patients with prior triptan insufficient efficacy and/or tolerability, efficacy with a higher dose (sumatriptan $100 \mathrm{mg}$ standard oral formulation) and/or alternative triptan formulation [rizatriptan $10 \mathrm{mg}$ ODT, sumatriptan $100 \mathrm{mg}$ rapid release, sumatriptan $6 \mathrm{mg}$ subcutaneous (SC)] was assessed. Pain freedom at $2 \mathrm{~h}$ was reported in (a) up to $61 \%$ of 
Table 3 Terminology and definitions used by RCTs and open-label studies to denote insufficient efficacy and/or tolerability to triptans

\begin{tabular}{|c|c|c|}
\hline Study & Terminology & Definition \\
\hline \multicolumn{3}{|c|}{ Previous insufficient efficacy and/or tolerability to triptans } \\
\hline \multicolumn{3}{|c|}{ Inadequate/unsatisfactory response } \\
\hline $\begin{array}{l}\text { Stark, } \\
2000[23]\end{array}$ & $\begin{array}{l}\text { Patients who "respond poorly" to oral } \\
\text { administration of sumatriptan }\end{array}$ & $\begin{array}{l}\text { Patients described themselves as experiencing an } \\
\text { unsatisfactory response to sumatriptan }\end{array}$ \\
\hline $\begin{array}{l}\text { Goldstein, } \\
2006[27]\end{array}$ & $\begin{array}{l}\text { Patients with previous "unsatisfactory response" to } \\
\text { rizatriptan }\end{array}$ & $\begin{array}{l}\text { Patients who were dissatisfied with their previous } \\
\text { treatment response to rizatriptan within the past } \\
12 \text { months, due to inadequate relief from } \\
\text { migraine headache pain; slow onset of migraine } \\
\text { pain relief; inadequate relief from associated } \\
\text { migraine symptoms (nausea, vomiting, } \\
\text { phonophobia, photophobia, etc.); recurrence of } \\
\text { migraine headache and/or needed to take } \\
\text { multiple doses of medication to keep the pain } \\
\text { away; delayed return to normal function; lack of } \\
\text { consistent response; troublesome side effects }\end{array}$ \\
\hline $\begin{array}{l}\text { Newman, } \\
2008[26]\end{array}$ & $\begin{array}{l}\text { Patients previously "dissatisfied" with sumatriptan } \\
\text { in any formulation at a dose lower than } 100 \mathrm{mg} \text {, } \\
\text { and had not received treatment with sumatriptan } \\
\text { at the } 100 \mathrm{mg} \text { dose prior to start of study }\end{array}$ & $\begin{array}{l}\text { Satisfaction was measured by a single-item question } \\
\text { with a 7-point response scale. Subjects who were } \\
\text { dissatisfied with previous sumatriptan and were } \\
\text { less than very satisfied with their current } \\
\text { treatment were included in the study }\end{array}$ \\
\hline \multirow[t]{2}{*}{$\begin{array}{l}\text { Brandes, } \\
2009[63]\end{array}$} & "Difficult-to-treat" menstrual migraine & $\begin{array}{l}\text { Difficult-to-treat menstrual migraine was defined as } \\
\text { having previous exposure to non-triptan therapy } \\
\text { for the treatment of menstrual migraine and an } \\
\text { inadequate response to triptan therapy for the } \\
\text { acute treatment of menstrual migraine over } \geq 2 \\
\text { menstrual cycles }\end{array}$ \\
\hline & & $\begin{array}{l}\text { An inadequate response to triptan therapy was } \\
\text { determined by the investigator using the Migraine } \\
\text { Medication History Questionnaire and defined as } \\
\text { a lack of efficacy or poor tolerability, triptan dose } \\
\text { in excess of the maximum recommended amount, } \\
\text { the need to use rescue medication, recurrence of } \\
\text { headache (within } 48 \mathrm{~h} \text { ), or partial response }\end{array}$ \\
\hline
\end{tabular}


Table 3 continued

\begin{tabular}{lll}
\hline Study & Terminology & Definition \\
\hline $\begin{array}{l}\text { Seeburger, } \\
2011[20]\end{array}$ & Sumatriptan "non-responders" & $\begin{array}{l}\text { Eligible participants reported that they did not } \\
\text { respond to treatment with sumatriptan and, at a } \\
\text { minimum, that they have demonstrated a } \geq 50 \% \\
\text { unsatisfactory response (e.g., no pain relief at } 2 \mathrm{~h} \\
\text { post dose) to sumatriptan across their total } \\
\text { migraine history (including failure to respond to } \\
\text { at least } 2 \text { administrations of sumatriptan) }\end{array}$
\end{tabular}

Discontinuation reasons

Farkkila, Patients with previous "poor response/tolerance" to Patients who had discontinued therapy with orally 2003 [22] oral administration of sumatriptan

Mathew, "Poor responders" intolerance to short-acting 2009 [29] triptans

Considered consistency of response

Mathew, Oral administration of sumatriptan "non-

$2000[25] \quad$ responders"

Landy, Patients with a history of "non-response" to

2004 [24] sumatriptan $50 \mathrm{mg}$

Diener, Patients with previous "poor response" to

2005 [21] sumatriptan $50 \mathrm{mg}$ administered sumatriptan between 2 weeks and 2 years prior to the screening visit. Subjects had been in the practices of the investigators for a significant period and their lack of enough response to sumatriptan was documented in the patient notes. Patients were asked to give one of the following reasons for stopping treatment with sumatriptan: slow onset of action; inconsistent response; poor overall efficacy; recurrence; tolerability

Poor response was defined as patient-reported discontinuation of treatment with a triptan for reasons related but not limited to slow onset of efficacy, inconsistent efficacy, poor overall efficacy, or poor sustained efficacy through $24 \mathrm{~h}$ or longer

Intolerance was defined as discontinuation of treatment with a triptan for any reason other than poor response, hypersensitivity, or allergic reaction

Defined as lack of response in at least $3 / 5$ attacks having taken the medication early in the headache phase ${ }^{a}$

Patients had a documented history of non-response to $50 \mathrm{mg}$ sumatriptan tablets at $2 \mathrm{~h}$ after dosing in the early, mild-pain phase of $2 / 3$ migraine attacks $^{a}$

Patients describing themselves as experiencing an unsatisfactory response to sumatriptan on $\geq 2$ prior occasions $^{\mathrm{a}}$ 
Table 3 continued

\begin{tabular}{ll}
\hline Study & Terminology \\
\hline $\begin{array}{c}\text { Diamond, } \\
2007[28]\end{array}$ & $\begin{array}{c}\text { Patients who historically "fail to respond" to orally } \\
\text { administered triptans }\end{array}$
\end{tabular}

\section{Definition}

Patients with migraine who historically had failed to achieve relief from an orally administered triptan in $2 / 3$ attacks. Relief was defined as reduction of pain severity from mild, moderate, or severe pain to no pain, or from moderate or severe pain to mild pain

Other

Ho,

$2011[31]$

Patients with self-reported historical triptan response of "poor/no use"
Knievel, "Non-responders" to triptan therapy 2018 [33]

Blumenfeld, Triptan "insufficient responder" 2019 [32]
Patients completed a migraine history questionnaire, which included the following question to determine whether patients had insufficient efficacy to triptans: "On average, how often did your moderate or severe migraine headaches respond to triptan medications?"

Patients had to select from $75-100 \%$ of the migraine attacks (good triptan historical response subgroup); $50-74 \%$ of the migraine attacks; $25-49 \%$ of the migraine attacks (intermediate triptan historical response subgroup); $<25 \%$ of the migraine attacks; you do not take triptans (poor/no historical triptan response subgroup); take triptans but do not know the frequency of response (excluded)

At baseline, patients rated themselves as good, poor, or non-responders to prior triptan therapy ${ }^{a}$

Patients were categorized on the basis of historical experience as a triptan-responder, triptaninsufficient responder, or triptan-naïve. Reasons for categorization as insufficient considered efficacy, tolerability, and contraindications or warnings ${ }^{\mathrm{a}}$

Insufficient efficacy and/or tolerability to triptans in a general population of patients with migraine

Cady, "Lack of response", "non-responders" $2007[35]$

Cady, 2014 "Non-responders"

[65]
Non-responders failed to meet endpoints "freedom from pain" at $2 \mathrm{~h}$ post dose, and $24 \mathrm{~h}$ "sustained freedom from pain”, and were permitted to use rescue medication $2 \mathrm{~h}$ post dose

Non-responders failed to meet the endpoint "mild or no pain $2 \mathrm{~h}$ post dose” without the use of a rescue medication or an increase in pain level within $24 \mathrm{~h}$ of treatment 
Table 3 continued

\begin{tabular}{|c|c|c|}
\hline$\overline{\text { Study }}$ & Terminology & Definition \\
\hline $\begin{array}{l}\text { Diener, } \\
2008[45]\end{array}$ & High risk of "non-response" & $\begin{array}{l}\text { Patients at high risk of non-response were identified } \\
\text { on the basis of a logistic regression analysis, using } \\
3 \text { predictors of not achieving a } 2 \text { h pain-free } \\
\text { response: severe baseline headache pain; } \\
\text { photophobia/phonophobia; nausea }\end{array}$ \\
\hline $\begin{array}{l}\text { Landy, } 2014 \\
{[66]}\end{array}$ & $\begin{array}{l}\text { Headache "non-responders", pain-free non- } \\
\text { responders }\end{array}$ & $\begin{array}{l}\text { Headache non-response at } 2 \mathrm{~h} \text { or sustained at } 24 \mathrm{~h} \text {, } \\
\text { defined by failing to experience a } 2 \text {-point } \\
\text { reduction in a } 4 \text {-point scale of pain intensity at } \\
2 \mathrm{~h} \text { or } 24 \mathrm{~h} \text {, respectively } \\
\text { Pain-free non-response at } 2 \mathrm{~h} \text { or sustained at } 24 \mathrm{~h} \text {, } \\
\text { defined by failing to achieve intensity of "no pain" }\end{array}$ \\
\hline $\begin{array}{l}\text { Miljkovic, } \\
2018[70]\end{array}$ & "Failure" of therapy & $\begin{array}{l}\text { Patients who failed to achieve complete reduction } \\
\text { of migraine pain } 2 \mathrm{~h} \text { post dose }\end{array}$ \\
\hline $\begin{array}{l}\text { Scott, } 1996 \\
{[64]}\end{array}$ & "Non-responders" & $\begin{array}{l}\text { Patients failed to meet the endpoint "headache } \\
\text { relief/freedom" in response to sumatriptan within } \\
4 \mathrm{~h} \text { of taking second dose }\end{array}$ \\
\hline $\begin{array}{l}\text { Spierings, } \\
2009[67]\end{array}$ & "Non-response" & $\begin{array}{l}\text { Patients who did not respond to frovatriptan } \\
\text { treatment within } 2-24 \text { h post dose }\end{array}$ \\
\hline $\begin{array}{l}\text { Tietjen, } \\
2005[34]\end{array}$ & "Inadequate relief", "not satisfied" & $\begin{array}{l}\text { Patients who had achieved (self-defined) } \\
\text { meaningful relief and whether they were satisfied } \\
\text { with naratriptan } 2.5 \mathrm{mg}\end{array}$ \\
\hline
\end{tabular}

$b$ hour

${ }^{a}$ Further objective criteria were not provided

b A limitation of this study was that this subgroup of patients comprised mainly (91\%) patients who reported that they did not take triptans, but it was not known whether these patients were triptan-naïve or had discontinued treatment with triptans

patients treated orally with sumatriptan $100 \mathrm{mg}$ rapid release, in participants dissatisfied with sumatriptan at a dose less than $100 \mathrm{mg}$ [26], (b) $80 \%$ of attacks treated with sumatriptan $100 \mathrm{mg}$ standard formulation in participants with non-response to sumatriptan $50 \mathrm{mg}$ [24], and (c) up to $56 \%$ of patients treated with sumatriptan $6 \mathrm{mg}$ SC in participants who had failed to respond to an orally administered triptan [28]. The study assessing rizatriptan $10 \mathrm{mg}$ ODT is described in an earlier section along with the other two studies that included a run-in validation phase [20].

\section{Combination Therapy in People with Insufficient Efficacy and/ or Tolerability to Triptans}

Three crossover studies investigated efficacy of combination treatment in patients who reported insufficient efficacy and/or tolerability to an orally administered triptan alone [29, 34]. Two were identical studies in patients who had discontinued a short-acting triptan and subsequently used an orally administered combination of sumatriptan $85 \mathrm{mg}$ plus naproxen $500 \mathrm{mg}$. Sumatriptan plus naproxen 
Table 4 Terminology and definitions used by observational studies to denote insufficient efficacy and/or tolerability to triptans

\begin{tabular}{|c|c|c|}
\hline Study & Terminology & Definition \\
\hline \multicolumn{3}{|c|}{ Historical insufficient efficacy and/or tolerability to triptans } \\
\hline \multicolumn{3}{|c|}{ Inadequate/unsatisfactory response } \\
\hline $\begin{array}{l}\text { Sarchielli, } \\
2006[38]\end{array}$ & "Non-responders" to rizatriptan & $\begin{array}{l}\text { Patients were selected on the basis of a poor } \\
\text { response to rizatriptan. Inefficacy was verified in } \\
\text { the treatment of at least } 3 \text { consecutive attacks } \\
\text { using the following definition: failure to achieve a } \\
\text { reduction in pain from severe or moderate to mild } \\
\text { or absence of pain within } 2 \mathrm{~h} \text { after rizatriptan } \\
\text { administration, without a recurrence in the next } \\
48 \mathrm{~h}\end{array}$ \\
\hline
\end{tabular}

Discontinuation reasons

Sheftell, Patients who were currently using a triptan as acute 2004 [68] treatment medication for migraine and who had

For each triptan/formulation used, information previously used at least one other triptan or a different triptan formulation regarding patients' satisfaction and reasons for discontinuation were summarized in following categories: to determine if another triptan is better; recurrence; incomplete/no relief; side effects; rebound; another triptan/formulation used was better; time to relief; other

Other

Silberstein, Triptan "insufficient responder" 2019 [37]

Patients currently prescribed triptans/had received triptans in the past 6 months and failed to achieve pain freedom at $2 \mathrm{~h}$, or discontinued because of lack of efficacy/side effects

Insufficient efficacy and/or tolerability to triptans in a general population

\begin{tabular}{|c|c|c|}
\hline $\begin{array}{l}\text { Alam, } \\
2018[72]\end{array}$ & Discontinued triptan users & $\begin{array}{l}\text { Assessed reasons for discontinuation from a pre- } \\
\text { coded list of side effects and triptan sensations }\end{array}$ \\
\hline $\begin{array}{l}\text { al Deeb, } \\
1997[69]\end{array}$ & "Poor or nil response" (and poor efficacy) & $\begin{array}{l}\text { A poor response to sumatriptan was characterized by } \\
\text { a drop of no points, operationalized on the basis } \\
\text { of pain diminution along a pain severity scale }\end{array}$ \\
\hline $\begin{array}{l}\text { Linder, } \\
1996[71]\end{array}$ & No response or "suboptimal response" & $\begin{array}{l}\text { Lack of efficacy defined by failing to meet the } \\
\text { endpoint "headache relief" }\end{array}$ \\
\hline $\begin{array}{l}\text { Munjal, } \\
2016[36]\end{array}$ & "Inadequate pain response" & $\begin{array}{l}\text { Patients who responded "never, rarely and less than } \\
\text { half the time" when asked if they achieved the } \\
\text { endpoint "pain freedom" at } 2 \mathrm{~h} \text { and " } 24 \mathrm{~h} \\
\text { sustained relief" }\end{array}$ \\
\hline
\end{tabular}


Table 4 continued

\begin{tabular}{|c|c|c|}
\hline Study & Terminology & Definition \\
\hline $\begin{array}{l}\text { Patrick, } \\
\text { 2000[39] }\end{array}$ & "Non-responders" & $\begin{array}{l}\text { Treatment response to the initial zolmitriptan dose } \\
\text { was assessed at } 2 \mathrm{~h} \text { using the following endpoints: } \\
\text { headache response, pain-free response, and } \\
\text { improvement in impairment of normal activity. } \\
\text { Patients also recorded whether they had obtained } \\
\text { meaningful migraine relief, a subjective global } \\
\text { evaluation of treatment response incorporating all } \\
\text { migraine symptoms, within } 2 \mathrm{~h} \text { of dose } \\
\text { administration. Non-responders were defined as } \\
\text { patients with a response rate of } 0-10 \%\end{array}$ \\
\hline $\begin{array}{l}\text { Peng, } \\
2016[40]\end{array}$ & "Non-responders" & $\begin{array}{l}\text { Patients were asked the effectiveness and AEs of one } \\
\text { sumatriptan tablet for their migraine attack; } \\
\text { however, non-response was not defined by the } \\
\text { authors }\end{array}$ \\
\hline $\begin{array}{l}\text { Seo, } \\
2016[41]\end{array}$ & Frovatriptan "inefficiency" & $\begin{array}{l}\text { Patients failed to meet the endpoint "pain relief or } \\
\text { absence" } 4 \mathrm{~h} \text { post dose in } 1 \text { of } 2 \text { successive } \\
\text { migraine attacks }\end{array}$ \\
\hline $\begin{array}{l}\text { Sheftell, } \\
2010[42]\end{array}$ & "Dissatisfied", non-responding headaches & $\begin{array}{l}\text { Secondary treatment failure was defined as a return } \\
\text { of pain to any level following initially successful } \\
\text { treatment }\end{array}$ \\
\hline $\begin{array}{c}\text { Terrazzino, } \\
2010[43]\end{array}$ & "Inconsistent response" & $\begin{array}{l}\text { Patients who did not experience a } \geq 2 \text {-point } \\
\text { reduction in a } 4 \text {-point scale of pain intensity } 2 \mathrm{~h} \\
\text { post dose in at least } 2 \text { out of } 3 \text { attacks }\end{array}$ \\
\hline $\begin{array}{l}\text { Wang, } \\
2017[44]\end{array}$ & "Non-responders" & $\begin{array}{l}\text { Patients who reported no response/effectiveness } \\
\text { based on the endpoint "freedom from pain, or } \\
\text { reduction to mild intensity in headache severity" } \\
\text { within } 2 \text { h post dose in at least } 2 \text { of } 3 \text { migraine } \\
\text { attacks }\end{array}$ \\
\hline
\end{tabular}

$A E$ adverse event, $b$ hour

was superior to placebo for pain freedom at $2 \mathrm{~h}$ (40-44\% vs. $14-17 \%)$ and sustained pain freedom $2-24 \mathrm{~h}(26-31 \%$ vs. 8\%) [29]. The third study investigated efficacy of orally administered naratriptan plus rectally administered prochlorperazine in patients with insufficient efficacy to orally administered naratriptan alone. None of the 14 patients who received this combination reported being pain-free at $2 \mathrm{~h}$ and the authors concluded that there was no evidence of benefit by adding prochlorperazine rectal suppository to naratriptan [34].

\section{Treatment of Patients with Allodynia}

Two studies identified in this SLR investigated triptan efficacy in patients with migraine with baseline allodynia or symptoms of subcutaneous sensitivity (SCS). In one study, pain freedom at $2 \mathrm{~h}$ with rizatriptan $10 \mathrm{mg}$, 
administered within $1 \mathrm{~h}$ of onset while pain was mild, ranged from $55 \%$ to $59 \%$ across patients both with and without baseline SCS [35]. At $2 \mathrm{~h}$ post dose, treatment with rizatriptan versus placebo led to significantly larger reductions in proportions of patients with SCS. The presence of SCS at time of dosing was not predictive of pain freedom at $2 \mathrm{~h}$; however, presence of SCS at $2 \mathrm{~h}$ correlated with lack of pain freedom at $2 \mathrm{~h}$ [35]. In the American Migraine Prevalence and Prevention (AMPP) study, in which medication was taken earlier in the course of attacks compared to most clinical trials, lack of pain freedom at $2 \mathrm{~h}$ in at least half of the attacks was reported more often in triptan users with allodynia (50\%) versus those without allodynia (36\%) [36].

\section{Characteristics Potentially Associated with Insufficient Efficacy and/ or Tolerability to Triptans}

Several observational studies investigated potential predictors and characteristics associated with triptan insufficient efficacy and/or tolerability [36-44]. In addition to those observational studies, two subgroup analyses from RCTs investigated predictors of insufficient efficacy within a single attack [35, 45].

Study participants with insufficient efficacy and/or tolerability to triptans were more likely to be younger $[40,41,44]$, of female gender $[40,42]$, and have clinical depression or have more symptoms of depression [36, 40, 41, 44]. Additionally, patients with triptan insufficient efficacy and/or tolerability commonly presented with more severe baseline headache intensity [36, 40, 42, 45], higher headache frequency $[36,44]$, baseline photophobia, phonophobia, and/or nausea [24, 29, 40, 42, 44, 45], presence of allodynia [36], and were less likely to be on migraine preventive medications $[36,43]$. It is not clear from the conference abstract if the timing for presence of allodynia was at time of dosing or over patients' lifetime [36].

Two studies identified that regular coffee consumption is associated with effectiveness of sumatriptan [40, 44]. One study showed that patients with a polymorphism in serotonin transporter gene were more likely to experience inconsistent response to a single triptan, as measured over three attacks [43]. Another study showed that insufficient efficacy to rizatriptan was correlated with lesser degree of trigeminal activation and lower variations of trigeminal neuropeptides after triptan administration [38]. Other studies showed that patients with triptan insufficient efficacy and/or tolerability were more likely to have higher opioid use, utilize a higher level of healthcare resources, have more rebound headaches, and have worse migrainespecific quality-of-life assessments [37, 39].

\section{DISCUSSION}

This comprehensive literature review identified substantial variability in methodology and definitions involving triptan insufficient efficacy and/or tolerability and focused on extracting clinically relevant evidence to provide value for clinicians and ultimately patients.

Definitions to identify patients with triptan insufficient efficacy commonly involved lack of pain freedom at $2 \mathrm{~h}$ and pain relief at $2 \mathrm{~h}$ in a single attack. For both outcomes, some definitions further included qualifiers such as achieving outcome over at least two out of three attacks. Some studies relied on prior discontinuation while others were purely subjective selfassessments based on treatment satisfaction. Several studies applied definitions that allowed for either efficacy or tolerability; none applied a definition specific only to tolerability.

The totality of evidence indicates that some people with insufficient response/tolerability to one triptan may benefit from switching to a different triptan. Switching studies that validated prior insufficient efficacy and/or tolerability to sumatriptan showed that approximately two-thirds of the participants did not experience pain relief at $2 \mathrm{~h}$ when rechallenged with sumatriptan within the trials $[20,21,23]$. In those studies, approximately $25-50 \%$ of patients subsequently experienced pain relief at $2 \mathrm{~h}$ with a different triptan. In studies that did not have a run-in validation period, up to two-thirds experienced pain relief 
at $2 \mathrm{~h}$ after subsequent treatment with a different triptan $[25,27,30]$. In two studies that specified early administration of a different oral triptan monotherapy, rates of pain freedom and pain relief at $2 \mathrm{~h}$ were relatively high compared to studies that did not treat early $[25,27]$.

None of the studies investigated subsequent outcomes in patients who had only triptan insufficient tolerability apart from those who had insufficient efficacy. A remaining unresolved question involves how many patients would benefit from a third triptan after failure to respond to an initial two triptans. Data from a claims database study showed that $8 \%$ of patients who initiated a triptan had at least three acute treatment changes (not specific to triptans) over a 1-year period [46]. However, none of the studies identified in this SLR assessed outcomes associated with three or more acute treatment changes.

Some studies identified here demonstrated benefit with a higher dose (sumatriptan $50 \mathrm{mg}$ to $100 \mathrm{mg}$ ) or different formulation (sumatrip$\tan$ rapid release, sumatriptan SC, rizatriptan ODT) of a triptan. Some identified benefit by adding a non-steroidal anti-inflammatory drug (NSAID). The combination of a triptan with an NSAID is recognized as a strategy for triptan insufficient efficacy in Canadian, French, German, the UK, and EHF treatment guidelines [47-51].

Terminology commonly used in studies included non-response or non-responder. However, those terms do not offer clear context as to whether the non-response refers to efficacy, tolerability, or both. In addition, lack of optimal response in these patients could be perceived as being a reflection of the patient rather than the treatment. It seems more accurate and empathetic to refer to "efficacy", "tolerability", or when not known (which is often the case), "efficacy and/or tolerability" when referring to patients experiencing insufficient response associated with treatment.

This review found that patients with triptan insufficient efficacy and/or tolerability were more likely to have more frequent and severe headaches; were more likely to have baseline photophobia, phonophobia, nausea, and allodynia; and were less likely to be on preventive medications or have regular caffeine intake. Additional studies have corroborated many of these predictors, especially with regard to higher severity of baseline pain, presence of nausea, and presence of photophobia or phonophobia [52, 53]. Some studies showed that patients in this population were more likely to be younger, female, and more likely to have signs of depression. Potential reasons could include that younger patients may have less experience with the natural history of migraine and with managing their individual attacks, and migraine attacks associated with menstruation may be more difficult to treat [54].

Insufficient response to triptans has been shown to result in considerable humanistic burden [55-57]. While effective for many, the unmet need remains high in patients using triptans, resulting in the need for newer drug classes $[58,59]$. With novel acute treatments emerging, however, the future algorithm for the acute treatment of migraine is not yet established. This includes guidance on the point at which switching to a different medication class should be considered. In the USA, the AHS has recently published a position statement regarding patients who have contraindications to triptans or who have failed to respond or tolerate at least two orally administered triptans. The statement recommends that these patients would be eligible for one of the new acute treatments, namely lasmiditan, ubrogepant, rimegepant, or neuromodulation device [1]. A recently published article not identified in this SLR, provides the response rates for lasmiditan versus placebo in patients who reported good and insufficient response to prior triptan therapy; efficacy was similar across these patient categories [60]. It may become clinically relevant to understand whether response rates of new treatments would be different in people with contraindications to triptans who immediately initiate new novel treatments versus those who have two prior triptan failures.

Increased physician awareness may help better identify patients at risk of insufficient response to triptans [55]. In addition to factors identified here, overuse of over-the-counter (OTC) medication and timing of triptan 
administration were factors identified in a global real-world study using data obtained from physicians and patients. Patient-Reported Outcomes (PROs) are mostly used in clinical research rather than in clinical practice. However, some PRO tools such as Migraine-Specific Quality of Life Questionnaire (MSQ) and Migraine Disability Assessment (MIDAS), although not specific to acute treatment, might give an idea of impact of attacks [55]. Further, it might be helpful to have some potential specific PRO tools such as Migraine Treatment Optimization Questionnaire (MTOQ) for the acute treatment which may aid physicians in identifying when to consider reassessment of migraine treatment options [55].

Strengths of this review include application of an up-to-date, rigorous systematic and comprehensive approach to identify all published studies in which the population of patients with triptan insufficient efficacy and/or tolerability was specifically assessed. Limitations include the selective use of published data, inclusion of only publications reported in English, and small numbers of patients in several studies. The design characteristics and statistical limitations of the identified studies have the potential to introduce inherent biases and lack validation. As a result of the heterogeneity and lack of uniformity across studies, results from this review are presented as a narrative synthesis without any comparisons across studies. Reliance on retrospective PROs, recall bias, and inter-subject variability also may have affected the findings. Within studies, besides the methodological limitations, there are some other plausible reasons that may contribute towards insufficient response to orally administered triptans including limited bioavailability and the impact of nausea and vomiting $[53,61]$. These factors vary between individuals and orally administered triptans. This may makes it difficult to assess true treatment failure rates, especially if a single attack is studied.

Several studies identified here did not apply a paradigm of early treatment that may influence their results and clinical interpretation. The importance of treating migraine attacks with triptans early and/or while headache severity is mild has been demonstrated in various studies, including the foundational "Act when Mild" study [62]. In clinical practice, patients may treat subsequent attacks earlier on the basis of personal experience. Also, given the variable characteristics of migraine treatments, there could be a learning curve when switching acute treatments. This learned behavior is likely to be associated with increased efficacy.

\section{CONCLUSIONS}

This compilation of results supports the assertion that a large unmet need remains in the acute treatment of migraine. Regardless of the methodologies or definitions applied across studies, a sizeable proportion of patients did not have optimal outcomes to subsequent treatment or strategies. Solutions to improve the management of those impacted could include increased patient and physician education, increased awareness of treatment optimization strategies, and availability of additional treatment options. Future studies can focus on prediction analyses using real-world data such as pharmacy databases to explore best possible treatment options for those with insufficient responses to triptans [58].

An increased level of consistency in defining insufficient efficacy could help identify patients who have the most potential to benefit from optimization of currently available treatments or availability of newly emerging acute treatments.

\section{ACKNOWLEDGEMENTS}

Funding. This research as well as journal's Rapid Service and Open Access Fees was funded by Eli Lilly and Company.

Medical Writing Assistance Editorial, and Other Assistance. Authors would like to thank Dr. Messoud Ashina for his suggestions on the article. Darren Joe (DJ) and Jack Said (JS), employees of Adelphi Values, were involved in executing the search and abstract screening for the project. Sriram Govindan and Richi Taneja, 
employees of Eli Lilly Service India Private Limited at the time of manuscript preparation, provided writing support. Uma Jyothi Kommoju, employee of Eli Lilly Services India Private Limited, provided writing support at the time of revision of the manuscript.

Authorship. All named authors meet the International Committee of Medical Journal Editors (ICMJE) criteria for authorship for this article, take responsibility for the integrity of the work as a whole, and have given their approval for this version to be published.

Disclosures. Andrew Buchanan, Louise Lombard, Li Shen Loo, and Brandy R Matthews are employees of Eli Lilly and Company and may be minor stockholders. Daisy Bridge, Ben Rousseau and Natasha Hopwood are employees of Adelphi Values. Elizabeth Leroux has received compensation for serving on advisory boards, speaking, and/or consulting from Allergan, Eli Lilly and Company, Novartis, Tribute Pharmaceuticals, McKesson Canada, Aralez Pharmaceuticals, Medscape and Teva Neurosciences; serves as president of the Canadian Headache Society. Uwe Reuter has received speaker fees and honorarium for consulting from Amgen, Allergan, Co-Lucid, Lilly Pharma, Medscape, Novartis, StreaMedUp, and TEVA Pharma.

Compliance with Ethics Guidelines. This article is based on previously conducted studies and does not contain any studies with human participants or animals performed by any of the authors.

Data Availability. All data generated or analyzed during this study are included in this published article/as supplementary information files.

Open Access. This article is licensed under a Creative Commons Attribution-NonCommercial 4.0 International License, which permits any non-commercial use, sharing, adaptation, distribution and reproduction in any medium or format, as long as you give appropriate credit to the original author(s) and the source, provide a link to the Creative Commons licence, and indicate if changes were made. The images or other third party material in this article are included in the article's Creative Commons licence, unless indicated otherwise in a credit line to the material. If material is not included in the article's Creative Commons licence and your intended use is not permitted by statutory regulation or exceeds the permitted use, you will need to obtain permission directly from the copyright holder. To view a copy of this licence, visit http:// creativecommons.org/licenses/by-nc/4.0/.

\section{REFERENCES}

1. American Headache Society. The American Headache Society position statement on integrating new migraine treatments into clinical practice. Headache. 2019;59(1):1-18.

2. Bartsch T, Knight YE, Goadsby PJ. Activation of $5-\mathrm{HT}(1 \mathrm{~B} / 1 \mathrm{D})$ receptor in the periaqueductal gray inhibits nociception. Ann Neurol. 2004;56(3): 371-81.

3. Tepper SJ, Rapoport AM, Sheftell FD. Mechanisms of action of the 5-HT1B/1D receptor agonists. Arch Neurol. 2002;59(7):1084-8.

4. Xia Y, Kelton CM, Wigle PR, Heaton PC, Guo JJ. Twenty years of triptans in the United States Medicaid programs: utilization and reimbursement trends from 1993 to 2013. Cephalalgia. 2016;36(14):1305-15.

5. Tfelt-Hansen P, Steiner TJ. Over-the-counter triptans for migraine: what are the implications? CNS Drugs. 2007;21(11):877-83.

6. Frisk P, Sporrong SK, Ljunggren G, Wettermark B, von Euler M. Utilisation of prescription and overthe-counter triptans: a cross-sectional study in Stockholm, Sweden. Eur J Clin Pharmacol. 2016;72(6):747-54.

7. Parkinson B, Gumbie M, Cutler H, Gauld N, Mumford V, Haywood P. Cost-effectiveness of reclassifying triptans in Australia: application of an economic evaluation approach to regulatory decisions. Value Health. 2019;22(3):293-302.

8. Viana M, Genazzani AA, Terrazzino S, Nappi G, Goadsby PJ. Triptan nonresponders: do they exist and who are they? Cephalalgia. 2013;33(11):891-6. 
9. Ferrari MD, Goadsby PJ, Roon KI, Lipton RB. Triptans (serotonin, 5-HT1B/1D agonists) in migraine: detailed results and methods of a meta-analysis of 53 trials. Cephalalgia. 2002;22(8):633-58.

10. Lipton RB, Buse DC, Serrano D, Holland S, Reed ML. Examination of unmet treatment needs among persons with episodic migraine: results of the American Migraine Prevalence and Prevention (AMPP) Study. Headache. 2013;53(8):1300-11.

11. Lipton RB, Hamelsky SW, Dayno JM. What do patients with migraine want from acute migraine treatment? Headache. 2002;42(Suppl 1):3-9.

12. Dodick DW. Triptan nonresponder studies: implications for clinical practice. Headache. 2005;45(2): 156-62.

13. Messali AJ, Yang M, Gillard P, et al. Treatment persistence and switching in triptan users: a systematic literature review. Headache. 2014;54(7): 1120-30.

14. Cameron C, Kelly S, Hsieh SC, et al. Triptans in the acute treatment of migraine: a systematic review and network meta-analysis. Headache. 2015;55(Suppl 4):221-35.

15. The Cochrane Collaboration. Cochrane handbook for systematic reviews of interventions version 5.1. 0. Updated March 2011. http://handbook. cochrane.org. Accessed Mar 19, 2020.

16. Wells GA, Shea B, O'Connell D, et al. The Newcastle-Ottowa Scale (NOS) for assessing the quality of nonrandomised studies in meta-analyses; 2019. http://www.ohri.ca/programs/clinical_ epidemiology/oxford.asp. Accessed Mar 19, 2020.

17. National Institute for Health and Clinical Excellence. Methods for development of NICE public health guidance (third edition); 2012. https://www. nice.org.uk/process/pmg4/chapter/introduction. Accessed Mar 19, 2020.

18. STROBE. STROBE checklist for cohort, case-control, and cross-sectional studies; 2009. https://www. strobe-statement.org/index.php?id=availablechecklists. Accessed Mar 19, 2020.

19. Moher D, Liberati A, Tetzlaff J, Altman DG, The PRISMA Group. Preferred reporting items for systematic reviews and metaanalyses: the PRISMA statement. PLoS Med. 2009;6(7):e1000097.

20. Seeburger JL, Taylor FR, Friedman D, et al. Efficacy and tolerability of rizatriptan for the treatment of acute migraine in sumatriptan non-responders. Cephalalgia. 2011;31(7):786-96.
21. Diener HC. Efficacy of almotriptan $12.5 \mathrm{mg}$ in achieving migraine-related composite endpoints: a double-blind, randomized, placebo-controlled study in patients controlled study in patients with previous poor response to sumatriptan $50 \mathrm{mg}$. Curr Med Res Opin. 2005;21(10):1603-10.

22. Farkkila M, Olesen J, Dahlof C, et al. Eletriptan for the treatment of migraine in patients with previous poor response or tolerance to oral sumatriptan. Cephalalgia. 2003;23(6):463-71.

23. Stark S, Spierings EL, McNeal S, Putnam GP, BoldenWatson CP, O'Quinn S. Naratriptan efficacy in migraineurs who respond poorly to oral sumatriptan. Headache. 2000;40(7):513-20.

24. Landy S, McGinnis J. Early migraine intervention with sumatriptan $100 \mathrm{mg}$ in patients with a history of nonresponse to sumatriptan $50 \mathrm{mg}$ : an open-label, prospective study of multiple attacks. Curr Ther Res Clin Exp. 2004;65(4):353-9.

25. Mathew NT, Kailasam J, Gentry P, Chernyshev O. Treatment of nonresponders to oral sumatriptan with zolmitriptan and rizatriptan: a comparative open trial. Headache. 2000;40(6):464-5.

26. Newman LC, Cady RK, Landy S, et al. Treatment satisfaction and efficacy of the rapid release formulation of sumatriptan $100 \mathrm{mg}$ tablets utilising an early intervention paradigm in patients previously unsatisfied with sumatriptan. Int J Clin Pract. 2008;62(12):1889-99.

27. Goldstein J, Tiseo PT, Albert KS, Li C, Sikes CR. Eletriptan in migraine patients reporting unsatisfactory response to rizatriptan. Headache. 2006;46(7):1142-50.

28. Diamond S, Freitag FG, Feoktistov A, Nissan G. Sumatriptan $6 \mathrm{mg}$ subcutaneous as an effective migraine treatment in patients with cutaneous allodynia who historically fail to respond to oral triptans. J Headache Pain. 2007;8(1):13-8.

29. Mathew NT, Landy S, Stark S, et al. Fixed-dose sumatriptan and naproxen in poor responders to triptans with a short half-life. Headache. 2009;49(7):971-82.

30. Färkkilä M, Diener H-C, Géraud G, et al. Efficacy and tolerability of lasmiditan, an oral 5-HT1F receptor agonist, for the acute treatment of migraine: a phase 2 randomised, placebo-controlled, parallel-group, dose-ranging study. Lancet Neurol. 2012;11(5):405-13.

31. Ho TW, Olesen J, Dodick DW, Kost J, Lines C, Ferrari MD. Antimigraine efficacy of telcagepant based on patient's historical triptan response. Headache. 2011;51(1):64-72. 
32. Blumenfeld AM, Goadsby PJ, Dodick DW, et al. Ubrogepant is effective for the acute treatment of migraine in patients with an insufficient response to triptans (P3.10-024). Neurology. 2019;92(15 Suppl):P3.10-024.

33. Knievel KLL, Buchanan A, et al. Response to lasmiditan for acute treatment of migraine based on prior response to triptan therapy. 12th European Headache Federation Congress jointly with 32nd National Congress of the Italian Society for the Study of Headaches, Florence, Italy. 28-30 September 2018. J Headache Pain. 2018;19(Suppl $1): 32-3$.

34. Tietjen GE, Athanas K, Utley C, Herial NA, Khuder $\mathrm{SA}$. The combination of naratriptan and prochlorperazine in migraine treatment. Headache. 2005;45(6):751-3.

35. Cady R, Martin V, Mauskop A, et al. Symptoms of cutaneous sensitivity pre-treatment and post-treatment: results from the rizatriptan TAME studies. Cephalalgia. 2007;27(9):1055-60.

36. Munjal S, Reed ML, Buse DC, Bennett A, Fanning $\mathrm{KM}$, Lipton $\mathrm{RB}$. The impact of triptan use and allodynia on initial and sustained response to medication in episodic migraine (EM): results from American migraine prevalence and prevention study (AMPP). In: Abstracts from the 58th Annual Scientific Meeting, AHS (June 9-16, 2016), 2016; PF04.2016; 56(S1):3-83.

37. Silberstein SD, Shewale AR, Baradaran S, et al. Opioid use, rebound headache, and resource utilization among migraine patients with insufficient response to triptans based on real-world data (S59.007). Neurology. 2019;92(15 Suppl):S59.007.

38. Sarchielli P, Pini LA, Zanchin G, et al. Clinical-biochemical correlates of migraine attacks in rizatriptan responders and non-responders. Cephalalgia. 2006;26(3):257-65.

39. Patrick DL, Hurst BC, Hughes J. Further development and testing of the migraine-specific quality of life (MSQOL) measure. Headache. 2000;40(7): 550-60.

40. Peng K, Fuh J, Wang S. The effectiveness and adverse events of sumatriptan: who are susceptible? In: Abstracts from the 58th Annual Scientific Meeting, AHS (June 9-16, 2016), 2016; PF04.2016; 56(S1):3-83.

41. Seo JG, Park SP. Factors associated with frovatriptan response in patients with migraine: a prospective, observational study. Cephalalgia. 2016;36(5): 493-8.
42. Sheftell F, Almas M, Weeks R, Mathew NT, Pitman $\mathrm{V}$, Lipton RB. Quantifying the return of headache in triptan-treated migraineurs: an observational study. Cephalalgia. 2010;30(7):838-46.

43. Terrazzino S, Viana M, Floriddia E, et al. The serotonin transporter gene polymorphism STin2 VNTR confers an increased risk of inconsistent response to triptans in migraine patients. Eur J Pharmacol. 2010;641(2-3):82-7.

44. Wang S-J, Peng K-P, Fuh J-L, Chen S-P, Wang Y-F. Sumatriptan response and predictors in migraine patients: a large clinic-based cohort study. Cephalalgia. 2017;37(1_suppl):25-51.

45. Diener HC, Dodick DW, Goadsby PJ, Lipton RB, Almas M, Parsons B. Identification of negative predictors of pain-free response to triptans: analysis of the eletriptan database. Cephalalgia. 2008;28(1): $35-40$.

46. Lombard L, Schroeder K, Nichols R, Kar-Chan Choong C, Ye W. Characteristics, treatment patterns, and healthcare resource utilization in patients with migraine who initiated a triptan. Headache. 2018;58(S2):182-183 [PS54].

47. Diener H-C, Holle-Lee D, Nägel S, et al. Treatment of migraine attacks and prevention of migraine: guidelines by the German Migraine and Headache Society and the German Society of Neurology. Clin Transl Neurosci. 2019;3(1):2514183X18823377.

48. Lanteri-Minet M, Valade D, Geraud G, Lucas C, Donnet A. Revised French guidelines for the diagnosis and management of migraine in adults and children. J Headache Pain. 2014;5:2.

49. National Institute for Health and Care Excellence. Headaches in over 12s: diagnosis and management. Clinical guideline [CG150]; 2012. https://www. nice.org.uk/guidance/cg150/chapter/Key-prioritiesfor-implementation. Accessed Mar 19, 2020.

50. Worthington I, Pringsheim T, Gawel MJ, et al. Canadian Headache Society Guideline: acute drug therapy for migraine headache. Can J Neurol Sci. 2013;40(5 Suppl 3):S1-80.

51. Steiner TJ, Jensen R, Katsarava Z, Linde M. Aids to management of headache disorders in primary care (2nd edition): on behalf of the European Headache Federation and Lifting The Burden: the Global Campaign against Headache. J Headache Pain. 2019;20(1):57.

52. Christoph-Diener H, Ferrari M, Mansbach H, SNAP Database Study Group. Predicting the response to sumatriptan: the Sumatriptan Naratriptan Aggregate Patient Database. Neurology. 2004;63(3): 520-4. 
53. Newman LC. Why triptan treatment can fail: focus on gastrointestinal manifestations of migraine. Headache. 2013;53(Suppl 1):11-6.

54. Maasumi K, Tepper SJ, Kriegler JS. Menstrual migraine and treatment options: review. Headache. 2017;57(2):194-208.

55. Lombard L, Farrar M, Ye W, et al. A global realworld assessment of the impact on health-related quality of life and work productivity of migraine in patients with insufficient versus good response to triptan medication. J Headache Pain. 2020;21(1):41.

56. Zebenholzer K, Gall W, Wöber C. Use and overuse of triptans in Austria-a survey based on nationwide healthcare claims data. J Headache Pain. 2018;19(1):34.

57. Piccinni C, Cevoli S, Ronconi G, et al. A real-world study on unmet medical needs in triptan-treated migraine: prevalence, preventive therapies and triptan use modification from a large Italian population along two years. J Headache Pain. 2019;20(1): 74.

58. Hansen TF, Chalmer MA, Haspang TM, Kogelman $\mathrm{L}$, Olesen J. Predicting treatment response using pharmacy register in migraine. J Headache Pain. 2019;20(1):31.

59. van Hoogstraten WS, Van Den Brink AM. The need for new acutely acting antimigraine drugs: moving safely outside acute medication overuse. J Headache Pain. 2019;20(1):54.

60. Knievel K, Buchanan AS, Lombard L, et al. Lasmiditan for the acute treatment of migraine: subgroup analyses by prior response to triptans. Cephalalgia. 2020;40(1):19-27.

61. Ferrari A, Tiraferri I, Neri L, Sternieri E. Why pharmacokinetic differences among oral triptans have little clinical importance: a comment. J Headache Pain. 2011;12(1):5-12.

62. Goadsby PJ, Zanchin G, Geraud G, et al. Early vs. non-early intervention in acute migraine-'Act when Mild (AwM)'. A double-blind, placebo-controlled trial of almotriptan. Cephalalgia. 2008;28(4): 383-91.

63. Brandes JL, Poole A, Kallela M, et al. Short-term frovatriptan for the prevention of difficult-to-treat menstrual migraine attacks. Cephalalgia. 2009;29(11):1133-48.
64. Scott RJ, Aitchison WR, Barker PR, McLaren GI. Oral sumatriptan in the acute treatment of migraine and migraine recurrence in general practice. QJM. 1996;89(8):613-22.

65. Cady R. CGRP, estrogen, cortisol, VIP, $\alpha$-amylase, PGE2, PGI2 and $\beta$-endorphin levels in menstrual migraine before and after treximet; 2014. https:// clinicaltrials.gov/ct2/show/NCT01329562. Accessed Mar 19, 2020.

66. Landy SH, Tepper SJ, Schweizer E, Almas M, Ramos E. Outcome for headache and pain-free nonresponders to treatment of the first attack: a pooled post hoc analysis of four randomized trials of eletriptan $40 \mathrm{mg}$. Cephalalgia. 2014;34(5):376-81.

67. Spierings EL, Keywood C. Rapid responders to frovatriptan in acute migraine treatment: results from a long-term, open-label study. Pain Med. 2009;10(4):633-8.

68. Sheftell FD, Feleppa M, Tepper SJ, Volcy M, Rapoport AM, Bigal ME. Patterns of use of triptans and reasons for switching them in a tertiary care migraine population. Headache. 2004;44(7):661-8.

69. al Deeb S, al Kawi Z, Yaqub B, Bohlega S, Cheung P. Sumatriptan treatment of acute migraine attacks in a Saudi population. Clin Neurol Neurosurg. 1997;99(1):23-5.

70. Miljkovic S, Smajlovic D, Crncevic S, et al. Efficient therapy for treatment of migraine without aura: ergotamine based drugs or sumatriptan. 12th European Headache Federation Congress jointly with 32nd National Congress of the Italian Society for the Study of Headaches. J Headache Pain. 2018;19(1):80.

71. Linder, S.L. Subcutaneous sumatriptan in the clinical setting: the first 50 consecutive patients with acute migraine in a pediatric neurology office practice. Headache J Head Face Pain 1996;36: 419-22.

72. Alam A, Munjal S, Reed ML. Triptan use and discontinuation in a representative sample of persons with migraine: results from Migraine in America symptoms and treatment (mast) study. Presented at 60th Annual Scientific Meeting American Headache Society® June 28-July 1, 2018 San Francisco Marriott Marquis San Francisco, CA. Headache J Head Face Pain. 2018;58(Suppl.2):68-69. 